\title{
Search for reference A0 dwarf stars: Masses and luminosities revisited with Hipparcos parallaxes ${ }^{\star, \star \star}$
}

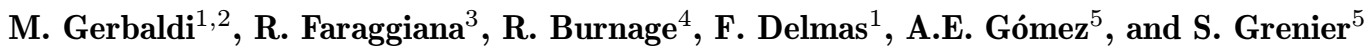 \\ 1 CNRS, Institut d'Astrophysique, 98bis, Bd. Arago, 75014 Paris, France \\ 2 Université de Paris-Sud XI, Paris, France \\ 3 Dipartimento di Astronomia, Università degli Studi di Trieste, v. Tiepolo 11, 34131 Trieste, Italy \\ 4 CNRS, Observatoire de Haute-Provence, 04870 Saint Michel l'Observatoire, France \\ 5 DASGAL, Observatoire de Paris-Meudon, Place Janssen, 92195 Meudon Cedex, France
}

Received November 12, 1998; accepted March 1, 1999

\begin{abstract}
Hipparcos data for 71 nearby dwarf A0 stars were combined with other data, in particular with high resolution spectra to establish the HR diagram in this temperature range. Almost $30 \%$ of unknown binaries were detected and discarded before establishing the $\mathcal{M}$-L relation for bright A0 $\mathrm{V}$ field stars. The relationship derived for these single stars is compared to the classical diagram derived from eclipsing binaries. The scatter of the latter is examined and the role of gravity is discussed.

A good agreement is found between the evolutionbased surface gravity $\log g_{\mathrm{ev}}$ and the value of $\log g_{\mathrm{ph}}$ obtained from photometric data.
\end{abstract}

Key words: stars: fundamental parameters — stars: early type — stars: HR diagram

\section{Introduction}

The access to precise Hipparcos parallaxes opens the possibility to go further in the knowledge of astrophysical stellar parameters when these parallaxes are coupled with other data.

The luminosity function of early-type stars allows to study the recent history of our Galaxy up to about 1 Gyr. We have selected the A0-type stars as representative of young objects which are long-lived enough on the main

Send offprint requests to: M. Gerbaldi

* Based on observations collected at the European Southern Observatory (ESO), La Silla, Chile in the framework of the Key Programme 5-004-43K and on data from the ESA Hipparcos astrometry satellite.

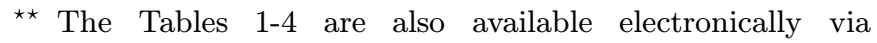
anonymous ftp 130.79 .128 .5 or via http://cdsweb.ustrasbg.fr/Abstract.html sequence to be present, in significant percentage, in the solar neighbourhood.

On one hand the use of field stars has the advantage, compared to cluster stars, to provide a larger sample of bright stars for which more accurate photometric and spectroscopic data can be obtained. On the other hand the use of field stars requires the knowledge of precise individual distances.

The present study is based on a sample of $71 \mathrm{~A} 0$ stars. This sample is extracted from a large set of data collected in the framework of an ESO Key Program (Gerbaldi et al. 1989), for studying B8 to F2 stars belonging to the Hipparcos Input Catalogue (Turon et al. 1992). These stars are classified non supergiant A0 in the Bright Star Catalogue (Hoffleit \& Jaschek 1982) [hereafter named $\mathrm{BSC}]$; for the few stars not belonging to the BSC, the spectral type is taken from the CDS data base. Stars classified as Ap and Am stars were discarded from the observational program. Among these 71 stars (limited to the Southern Hemisphere) 50 are classified A0 V and represent $21 \%$ of the BSC A0 V stars; these stars are expected to be at a distance $d \leq 150 \mathrm{pc}$.

The purpose of this study is to select a reference sample of non supergiant A0 stars to be used for the construction of an accurate HR diagram where $M_{V}$ is derived from the Hipparcos parallaxes.

The spectroscopic observations are used to verify, through the comparison between observed and computed spectra, the accuracy of the photometrically derived atmospheric parameters; several criteria are used to detect possible peculiarities.

The determination of $T_{\text {eff }}$ and $\log g$ is discussed in Sect. 3 ; the computations of the grid of synthetic spectra and the fit on the observed ones are described in Sect. 4 ; the results obtained from the spectral analysis are 
presented in Sects. 5 and 6. A subsample of non binary, non peculiar stars is defined (Sect. 7) for which the resulting HR diagram is discussed in Sect. 8.1; in Sect. 8.2 the gravity derived from atmospheric properties (photometric and spectroscopic data) are compared with that obtained combining stellar evolution models and luminosity computed from the Hipparcos parallaxes. In Sect. 8.3 the Mass-Luminosity relationship is analyzed and compared to the empirical one, derived from the eclipsing binaries.

\section{Observations and data reduction}

The basic data on the observed stars have been gathered in Table 1. Star identifications are given in Cols. 1 and 2, the spectral types from the BSC and from Abt and Morrell (1995) (hereafter AM) in Cols. 3 and 4.

The list of the established double stars in this sample can be extracted from the informations given in Annex 1 of the Hipparcos Input Catalogue (Turon et al. 1992), as well as from the Hipparcos and Tycho Catalogues (ESA, 1997) for the new ones discovered during this mission. For visual doubles the magnitude difference and the angular separation, given in Cols. 5 and 6 , are for the systems for which these values can affect the measures. These informations are given in the last column of Table 1. Results from search for binarity by speckle observations (Col. 7) are taken from the update of the Bright Star Catalogue (Hoffleit \& Warren 1994) [hereafter named BSC1994], kindly communicated by W.H. Warren. Projected rotational velocities, according to the BSC and to AM are added in Cols. 8 and 9 .

The spectra were obtained with the ECHELEC spectrograph mounted at the ESO $1.5 \mathrm{~m}$ telescope, in the period Jan. 89 - Jan. 95. The linear dispersion is about $3.1 \AA \mathrm{mm}^{-1}$ in the chosen wavelength range $4210-4500 \AA$. The slit width of $320 \mu \mathrm{m}$ corresponds to 1.52 arcsec on the sky. From unblended thorium lines, the FWHM of the instrumental profile was measured; its value is $0.17 \AA$, in agreement with the size of the entrance slit. The original frame covers 11 orders of the echelle spectrum; only 9 of them have been retained and reduced, while the 2 truncated orders on both sides of the central wavelength have been discarded. Flat field correction with a tungsten lamp and wavelength calibration with a Th lamp have been made with classical procedures. Great care was devoted to remove instrumental defects which arose from time to time: the most important ones were the two ghosts present as transversal bars on the original frames in the 1991-1994 period; the method is based on the accurate analysis of the intensity in the inter-orders. The resolution is about 28000 and the $\mathrm{S} / \mathrm{N}$, highly variable from the center to the edges of each order, covers the range 50 to 200.

The analysis of the $\mathrm{H}_{\gamma}$ profile being one of the goals of the present study, we had to produce a single spectrum by interconnecting the echelle orders. A particular attention was given to the method adopted for this connection. The complete reduction procedure is described in Burnage \& Gerbaldi (1990, 1992). The broad spectral domain covered by the $\mathrm{H}_{\gamma}$ line in $\mathrm{A} 0$ dwarf stars imposes to give a particular attention to the procedure adopted to normalize the spectra. The normalization is made by trial and error method; first the spectra have been normalized in the classical way by drawing the continuum through the highest points of the spectrum and then overplotted on a template synthetic spectrum. This allows to choose a number of continuum points common to both; these points are used as references in the final normalization procedure.

Since the observations span over 5 years, we have systematically observed four stars. The number of spectra obtained for each of them are: HD 15371: 19 spectra, HD 48915 (Sirius): 31 spectra, HD 149348: 13 spectra, HD 193924: 15 spectra. We checked the instrumental stability of the spectrograph and the consistency of the reduction procedures through the comparison of the spectra of these stars.

The reliability of the photometric calibration given by the flat field correction has been investigated through the measurement of the equivalent width (hereafter written EW) of lines selected over the observed spectral range; the number of lines measured in each spectrum was: 9 for HD 15371, 29 for HD 48915, 15 for HD 149348 and 6 for HD 193924. For each of these stars, we controlled that the distribution of the EWs of the selected lines is Gaussian; the mean value of the EW for each of these lines has been computed and the mean value of all the standard deviation is smaller than $0.005 \AA$.

The mechanical stability of the instrument has been tested through the measurement of the position of the same set of lines in the same stars. The final result is that the rms of line wavelengths corresponds to $1 \mathrm{~km} \mathrm{~s}^{-1}$.

We verified also, using all the spectra of HD 48915, that the profile shape, after the normalization of the spectra, is conserved whatever the setup of the spectrograph has been.

These necessary tests allow us to use confidently the whole set of observations as an homogeneous sample of data in the following analysis.

\section{Determination of $T_{\text {eff }}, \log g$}

For large samples of stars, the best method for $T_{\text {eff }}, \log g$ determination is based on the use of calibrated photometric indices. For early-type stars the $u v b y \beta$ and Geneva photometric systems are the most commonly used. In the $u v b y \beta$ photometric system specific filters measure the strength of the $\mathrm{H} \beta$ line.

We have determined $T_{\text {eff }}$ and $\log g$ using calibrations of both photometric systems. A non systematic discrepancy 
Table 1. The programme stars

\begin{tabular}{|c|c|c|c|c|c|c|c|c|c|}
\hline HD & $\mathrm{HR}$ & $\mathrm{Sp}(\mathrm{BSC})$ & $\mathrm{Sp}(\mathrm{AM})$ & $\Delta m$ & $\begin{array}{c}\text { Sep } \\
\text { arcsec }\end{array}$ & $\begin{array}{c}\text { Speckle } \\
\text { arcsec }\end{array}$ & $\begin{array}{c}\text { Rot(BSC) } \\
\mathrm{km} \mathrm{s}^{-1}\end{array}$ & $\begin{array}{c}\text { Rot(AM) } \\
\mathrm{km} \mathrm{s}^{-1}\end{array}$ & Remarks \\
\hline 3003 & 136 & $\mathrm{~A} 0 \mathrm{~V}$ & A1 IV & 0.2 & 0.1 & & 84 & & \\
\hline 4065 & 185 & A0 V & - & 0.3 & 0.7 & 0.731 & - & - & Hipparcos $0.43 \mathrm{mag} 0.737 \operatorname{arcsec}$ \\
\hline 4150 & 191 & A0 IV & A0 IV & & & & - & $\ldots$ & \\
\hline 7916 & 380 & $\mathrm{~A} 0 \mathrm{~V}$ & & 2.4 & 2.4 & & - & - & Hipparcos $1.95 \mathrm{mag} 2.435 \operatorname{arscsec}$ \\
\hline 15004 & 704 & A0 III & B9 III:n + shell (HI) & & & & - & 200 & \\
\hline 15646 & 734 & A $0 \mathrm{~V}$ & & & & & - & - & \\
\hline 16152 & - & A0 & & & & & - & - & \\
\hline 17864 & 853 & A $0 \mathrm{~V}$ & & & & & - & - & Hip. new binary $3.82 \mathrm{mag} 0.314 \operatorname{arcsec}$ \\
\hline 18735 & 903 & A0 $\mathrm{V}$ & & & & & - & - & \\
\hline 20980 & 1018 & A $0 \mathrm{~V}$ & $\mathrm{~A} 1 \mathrm{~V}$ & & & & - & 35 & \\
\hline 21473 & 1049 & A $0 \mathrm{~V}$ & & & & & - & - & \\
\hline 22789 & 1114 & A $0 \mathrm{~V}$ & A $0 \mathrm{~V}$ & & & & - & 135 & \\
\hline 27660 & - & A0 & & & & & - & - & \\
\hline 30397 & 1524 & A $0 \mathrm{~V}$ & & & & & - & - & \\
\hline 31295 & 1570 & A $0 \mathrm{~V}$ & A0 Vp $(\lambda$ Boo $)$ & & & & 104 & 105 & \\
\hline 32996 & 1661 & $\mathrm{~A} 0 \mathrm{~V}$ & A1 Vp (Si st,Ca wk) & & & & $\leq 41$ & 15 & \\
\hline 34868 & 1758 & A0 V & A1 IV & & & & 69 & 90 & \\
\hline 34968 & 1762 & $\mathrm{~A} 0 \mathrm{Vv}$ & B9 III & 3.8 & 4.2 & & 51 & 70 & Hipparcos $3.72 \mathrm{mag} 4.108 \operatorname{arcsec}$ \\
\hline 36473 & 1849 & A0 V & A $0 \mathrm{~V}$ & & & & 60 & 45 & \\
\hline 38056 & 1966 & A $0 \mathrm{~V}$ & & & & & - & - & \\
\hline 38206 & 1975 & $\mathrm{~A} 0 \mathrm{Vs}$ & A0 IIIs & & & negative & $\leq 41$ & 25 & \\
\hline 42301 & 2180 & A0 IV & A0 Vn & & & & - & 225: & \\
\hline 42729 & 2206 & A $0 \mathrm{~V}$ & B9.5 V & & & & - & 20 & \\
\hline 42834 & 2211 & $\mathrm{~A} 0 \mathrm{~V}$ & & & & & - & - & \\
\hline 45557 & 2345 & A0 $\mathrm{V}$ & $\mathrm{A} 0 \mathrm{~V}$ & & & & - & & \\
\hline 47827 & 2455 & A0 & B9.5 Vp (4481 wk) & 0.4 & 0.1 & $0.058^{*}$ & - & 15 & $*=$ speckle from Hartkopf et al. $(1996)$ \\
\hline 56341 & 2755 & A0 & A0 V & & & & - & 35 & \\
\hline 60629 & 2912 & $\mathrm{~A} 0 \mathrm{~V}$ & A0.5 III & & & & - & 40 & \\
\hline 63112 & 3019 & A0 V & & & & negative & $\leq 41$ & - & \\
\hline 63584 & 3038 & A0 IV-V & & & & & - & - & \\
\hline 67725 & 3189 & A0 Vn & A0 Vp (4481 wk) & & & negative & 160 & $\ldots$ & \\
\hline 69589 & 3255 & A0 & A1 IV & & & & - & 110 & \\
\hline 71043 & 3300 & A $0 \mathrm{~V}$ & & & & & - & - & \\
\hline 71155 & 3314 & A0 V & A0 $\mathrm{V}$ & & & negative & 125 & 115 & \\
\hline 71576 & 3334 & A0-1 IV-V & & & & & - & - & \\
\hline 74475 & 3463 & A0 $\mathrm{V}$ & & & & & - & - & \\
\hline 76346 & 3549 & A $0 \mathrm{~V}$ & & & & & - & - & \\
\hline 79108 & 3651 & $\mathrm{~A} 0 \mathrm{~V}$ & A0 Vp $(\lambda$ Boo $)$ & & & negative & 150 & 160 & \\
\hline 80950 & 3721 & A $0 \mathrm{~V}$ & & & & & - & - & \\
\hline 84461 & 3875 & A0 IV & & & & & 16 & - & \\
\hline 85504 & 3906 & $\mathrm{~A} 0 \mathrm{Vs}$ & B9.5 III & & & negative & $\leq 41$ & 15 & \\
\hline 87344 & 3963 & A0 V & B9 IV & & & negative & 17 & 15 & \\
\hline 87363 & 3964 & $\mathrm{~A} 0 \mathrm{~V}$ & & & & & - & - & \\
\hline 87887 & 3981 & A0 III & B9.5 III & & & & 9 & 10 & \\
\hline 92845 & 4194 & A0 V & & & & & 131 & - & \\
\hline 97585 & 4356 & $\mathrm{~A} 0 \mathrm{~V}$ & B9.5 Vn & & & negative & 177 & 175 & \\
\hline 99922 & 4428 & A $0 \mathrm{~V}$ & & 3.0 & 8.3 & & - & - & Hipparcos $3.31 \mathrm{mag} 8.185 \operatorname{arcsec}$ \\
\hline 101615 & 4502 & A0 V & & & & & 0 & - & \\
\hline 104039 & 4579 & A0-1 V & A1 IIIs & 0.2 & 0.2 & & 0 & $<10$ & \\
\hline 104430 & 4592 & A0 V & & & & & - & - & \\
\hline 106797 & 4669 & A $0 \mathrm{~V}$ & & & & & - & - & \\
\hline 109573 & 4796 & A0 & & & & negative & - & - & \\
\hline 111519 & 4871 & $\mathrm{~A} 0 \mathrm{~V}^{*}$ & & & & & - & - & $*=\mathrm{A} 0 \mathrm{Vm}(\mathrm{BSC}, 1994)$ \\
\hline 111786 & 4881 & A0 III & F0 Vp ( $\lambda$ Boo,met:A1) & & & & - & 135 & \\
\hline 113852 & 4947 & A0 IV-III & & & & & $\leq 49$ & - & \\
\hline 114570 & 4977 & A0 Vm: & & & & & ${ }_{-}^{-}$ & - & \\
\hline 125473 & 5367 & A0 IV & B9.5 V & & & & 101 & $\ldots$ & \\
\hline 129791 & - & A0 V & & & & & - & - & \\
\hline 139129 & 5798 & A0 V & & & & & 0 & - & \\
\hline 151527 & 6235 & A0 Vn & B9.5 Vp (4481wk)n & & & negative & 139 & 225: & \\
\hline 152849 & 6291 & $\mathrm{~A} 0 \mathrm{~V}$ & A0 Vn & 0.3 & 1.1 & 0.993 & - & 190 & Hipparcos $0.35 \mathrm{mag} 0.998 \operatorname{arcsec}$ \\
\hline 160263 & 6572 & A $0 \mathrm{~V}$ & & & & & - & - & \\
\hline 170680 & 6944 & A0 $\mathrm{Vn}^{*}$ & A0 $\mathrm{Vp}(\lambda \mathrm{Boo})$ & & & negative & 213 & 200: & $*=\mathrm{A} 0 \mathrm{Vp}(\mathrm{BSC}, 1994)$ \\
\hline 188228 & 7590 & $\mathrm{~A} 0 \mathrm{~V}$ & A0 IV & & & & 110 & & \\
\hline 193571 & 7779 & A0 V & & & & negative & - & - & \\
\hline 213320 & 8573 & A0 IVs & A0 III & & & & 23 & 10 & Hip. new binary $3.60 \mathrm{mag} 3.744 \operatorname{arcsec}$ \\
\hline 213398 & 8576 & A0 V & A1 III & & & negative & 36 & $\ldots$ & \\
\hline 216931 & - & A0 & & & & & - & - & \\
\hline 218639 & 8816 & A0 Vn & $\mathrm{A} 0 \mathrm{Vn}$ & & & & 154 & 235: & \\
\hline 223352 & 9016 & A0 V & A0 $\mathrm{Vp}(\lambda \mathrm{Boo}) \mathrm{n}$ & & & & - & 280: & \\
\hline 225200 & 9102 & $\mathrm{~A} 0 \mathrm{~V}$ & B9 IVs + A2n & & & & - & 315: & \\
\hline
\end{tabular}


between the the two sets of values will be used as an additional information to interpret the spectra. We adopted the Moon \& Dworetsky (1985) [hereafter MD] and Künzli et al. (1997) calibrations for uvby $\beta$ and Geneva systems respectively.

The homogenized uvby $\beta$ colours are taken from the Hauck \& Mermilliod (1990) Catalogue [hereafter HM] and are given in Cols. 2 to 5 of Table 2 . In this catalogue the Strömgren indices are erroneous for HD 225200 (Geneva code $=409010021$ ) and we adopted the values from the Simbad data base. The indices are missing for the 2 stars HD 27660 and HD 216931 and we checked that there is no update in the recent edition by Mermilliod et al. (1997). The reddening of these stars has been estimated from the $U B V$ indices: it is negligible for the first star and $E(B-V)=0.06$ is derived for the second one. We also note that the secondary component of the broad visual double HD 87344, indicated as HD 87344(2) $(V=$ 8.0), in the Hauck and Mermilliod Catalogue is in reality HD $87330(V=7.13$, B9III-IV). Its spectrum clearly shows that the star HD 87330 is an SB2 with a well developed system of double lines at the epoch of our observations (May 13, 1990 and April 8, 1993).

For the Geneva photometry the version of the Catalogue available at CDS has been used. HD 129791 is not included in this Catalogue.

We recall that as a first step dereddened colours must be computed; for our sample of stars, dereddening is expected to be very low or negligible; a strong reddening is likely to indicate a flux distortion due to spectral peculiarities or undetected binarity.

\subsection{Reddening and its correction}

For these bright stars, mainly belonging to the BSC, standard methods of dereddening are assumed to be valid, these stars being expected to be slightly reddened. We used the programs by Moon (1985) to compute the colour excess. A similar determination cannot be performed from the Geneva photometric indices because no updated procedures are published.

The A-type stars are in the domain where the Balmer lines reach their maximum. According to Strömgren (1966), the uvby $\beta$ calibration requires that each star should be assigned to one of the three groups: early ( $\beta$ depends mainly on the luminosity, $c_{0}$ is mainly related to the $T_{\text {eff }}$ ), late (the roles of $\beta$ and $c_{0}$ are reversed) or intermediate (for which a combination of various indices must be used). In the program UVBYBETA by Moon (1985) the choice of the group is based on photometric quantities and spectral classification. Since the boundaries between the groups overlap in some cases, the assignment to a group is sometimes ambiguous and uncertainty in group selection cannot be avoided. In this case, the computations have been made for both groups; the result is an average difference of 0.02 in the colour excess $E(b-y)$. Details concerning this effect can be found in Gerbaldi et al. (1998a). The final choice of the group defined in the UVBYBETA program is given in Col. 6 of Table 2 ; this choice is made on the basis of the lower $E(b-y)$ and by the analysis of the atmospheric parameters derived for both cases (see next subsection).

The determination of the amount of reddening, being based on the empirical calibration of the uvby $\beta$ system, is model independent. However, the intrinsic colours are slightly different according to different authors and this may lead to different values of the colour excess; a discussion on this effect can be found in Figueras et al. (1991) and in Jordi et al. (1997). According to these papers, for our sample of nearby stars, we can safely use the Moon program UVBYBETA without any further modification.

The value of $E(b-y)$ is given in Col. 7 of Table 2 . Only 6 stars have $E(b-y)$ higher than 0.02 ; to this we can add the estimated value for HD 216931. The highest value, $E(b-y)=0.05$, is found only in one case, for HD 151527, which with HD $111786[E(b-y)=0.00]$ are the only two stars to present the peculiarity of a $(b-y)$ index very high for stars belonging to the A0 type. These two newly detected binaries are further discussed below. For the three stars HD 7916, HD 114570 and HD 129791 the colour excess is $E(b-y)=0.04$; for two others HD 67725 , HD 104039 the value of the colour excess is $E(b-y)=0.03$.

For the remaining stars the $E(b-y)$ is what expected, i.e. in the range $-0.01,+0.02$, with the exception of HD 60629, which has a slightly higher "blueing" $E(b-y)=-0.02$. These values of reddening and bluing are interpreted as possible sign of abnormality. These stars will be discussed in Sect. 7 .

\section{2. $T_{\mathrm{eff}}$ and $\log g$ from uvby $\beta$ photometry}

The atmospheric parameters have been derived from the uvby $\beta$ photometry, using the calibration by Moon \& Dworetsky (1985) (Table 2, Cols. 8 and 9) which has been tested by recent studies (Napiwotzki et al. 1993; Smalley \& Dworetsky 1993, for stars cooler than A0). The corrections determined by Castelli (1991) and by Dworetsky \& Moon (1986) have no influence on our range of parameters.

The internal errors on $T_{\text {eff }}$ and $\log g$ due to the scatter of the individual observed colours have been estimated by Lemke (1989) to be approximately $\pm 100 \mathrm{~K}$ and \pm 0.1 dex respectively. A different approach to the evaluation of these errors is found in Gerbaldi et al. (1998b), where it is shown that a difference of 0.015 in colour excess implies a difference of $200 \mathrm{~K}$ in $T_{\text {eff }}$. 
Table 2. Photometric, astrometric data and atmospheric parameters

\begin{tabular}{|c|c|c|c|c|c|c|c|c|c|c|c|c|c|c|c|c|}
\hline HD & $b-y$ & $m_{1}$ & $c_{1}$ & $\beta$ & Gr & $E(b-y)$ & \multicolumn{2}{|c|}{$\begin{array}{c}T_{\text {eff }} \log g \\
\text { MD }\end{array}$} & \multicolumn{2}{|c|}{$\begin{array}{c}T_{\text {eff }} \log g \\
\text { Gen }\end{array}$} & Broad & \multicolumn{2}{|c|}{$\operatorname{mas}^{\frac{\sigma \pi}{\pi}}$} & $M_{v}$ & $\sigma$ & HIP \\
\hline 3003 & 0.014 & 0.179 & 0.991 & 2.910 & 5 & -0.01 & 9340 & 4.38 & 9231 & 4.19 & 84 & 21.52 & 0.02 & 1.74 & 0.05 & 2578 \\
\hline 4065 & -0.019 & 0.153 & 0.909 & 2.865 & 1 & 0.00 & 10550 & 4.28 & 10242 & 4.16 & 30 & 7.55 & 0.16 & 0.44 & 0.35 & 3356 \\
\hline 4150 & 0.012 & 0.133 & 1.090 & 2.858 & 1 & 0.02 & 9810 & 3.71 & & & 130 & 13.57 & 0.04 & -0.06 & 0.09 & 3405 \\
\hline 7916 & 0.036 & 0.150 & 0.967 & 2.870 & 5 & 0.04 & 9830 & 4.17 & & & 200 & 9.69 & 0.06 & 0.99 & 0.13 & 5992 \\
\hline 15004 & 0.007 & 0.109 & 1.065 & 2.786 & 1 & 0.02 & 10220 & 3.29 & & & 200 & 5.05 & 0.21 & -0.23 & 0.46 & 11261 \\
\hline 15646 & -0.014 & 0.150 & 1.027 & 2.889 & 5 & 0.00 & 10020 & 4.13 & 9974 & 4.08 & 50 & 8.47 & 0.06 & 0.99 & 0.14 & 11479 \\
\hline 16152 & 0.007 & 0.173 & 0.995 & - & 5 & -0.01 & 9510 & 4.36 & 9598 & 4.24 & 200 & 8.15 & 0.11 & 1.67 & 0.24 & 12055 \\
\hline 17864 & 0.015 & 0.169 & 1.006 & 2.894 & 5 & 0.00 & 9420 & 4.23 & 9174 & 4.02 & 200 & 10.02 & 0.08 & 1.37 & 0.17 & 13271 \\
\hline 18735 & 0.001 & 0.159 & 1.067 & 2.899 & 5 & 0.00 & 9590 & 4.09 & 9464 & 4.00 & 170 & 7.90 & 0.08 & 0.80 & 0.17 & 13947 \\
\hline 20980 & -0.001 & 0.165 & 1.042 & 2.916 & 5 & 0.00 & 9590 & 4.29 & 9431 & 4.18 & 30 & 7.25 & 0.10 & 0.66 & 0.22 & 15700 \\
\hline 21473 & 0.022 & 0.170 & 1.110 & 2.895 & 5 & 0.01 & 9070 & 3.93 & 8895 & $\begin{array}{l}3.90 \\
3.96\end{array}$ & 50 & 7.39 & 0.07 & 0.63 & 0.16 & 16015 \\
\hline 22789 & -0.020 & 0.141 & 1.068 & 2.858 & 1 & -0.01 & 10110 & 3.76 & 9881 & 3.87 & 100 & 7.81 & 0.09 & 0.47 & 0.21 & 17007 \\
\hline 27660 & & & & & & & & & 8594 & 3.40 & 200 & 6.54 & 0.13 & 0.48 & 0.28 & 20360 \\
\hline 30397 & 0.001 & 0.151 & 1.088 & 2.896 & 5 & 0.01 & 9700 & 4.01 & 9286 & 3.84 & 50 & 4.67 & 0.14 & 0.15 & 0.30 & 22136 \\
\hline 31295 & 0.044 & 0.178 & 1.007 & 2.898 & 5 & 0.01 & 8970 & 4.25 & 8763 & 4.18 & 100 & 27.04 & 0.03 & 1.76 & 0.08 & 22845 \\
\hline 32996 & -0.019 & 0.140 & 0.939 & 2.848 & 1 & 0.00 & 10520 & 4.05 & 10364 & 3.99 & 20 & 8.98 & 0.08 & 0.81 & 0.18 & 23777 \\
\hline 34868 & -0.017 & 0.141 & 1.043 & 2.880 & 5 & 0.00 & 10120 & 4.01 & 9983 & 3.87 & 100 & 7.31 & 0.08 & 0.30 & 0.18 & 24831 \\
\hline 34968 & -0.020 & 0.124 & 1.059 & 2.830 & 1 & -0.01 & 10250 & 3.59 & 10160 & 3.67 & 70 & 7.11 & 0.09 & -1.04 & 0.20 & 24927 \\
\hline 36473 & 0.003 & 0.155 & 1.038 & 2.899 & 5 & 0.01 & 9740 & 4.18 & 9605 & 4.10 & 50 & 12.00 & 0.07 & 0.89 & 0.14 & 25853 \\
\hline 38056 & -0.020 & 0.143 & 0.965 & 2.864 & 1 & 0.00 & 10400 & 4.11 & 10358 & 4.07 & 200 & 7.55 & 0.07 & 0.74 & 0.16 & 26796 \\
\hline 38206 & -0.014 & 0.165 & 0.940 & 2.907 & 5 & -0.01 & 10170 & 4.48 & 10174 & 4.36 & 30 & 14.45 & 0.05 & 1.53 & 0.11 & 26966 \\
\hline 42301 & -0.008 & 0.146 & 1.030 & 2.877 & 1 & 0.00 & 9990 & 4.02 & 9856 & 4.07 & $200-300$ & 12.77 & 0.05 & 1.02 & 0.12 & 29150 \\
\hline 42729 & -0.009 & 0.127 & 1.131 & 2.847 & 1 & 0.00 & 9760 & 3.54 & 9702 & 3.61 & 30 & 4.83 & 0.13 & -0.50 & 0.27 & 29347 \\
\hline 42834 & -0.006 & 0.123 & 1.113 & 2.833 & 1 & 0.00 & 9840 & 3.51 & 9670 & 3.61 & 150 & 4.92 & 0.10 & -0.26 & 0.22 & 29304 \\
\hline 45557 & -0.011 & 0.166 & 1.031 & 2.890 & 5 & -0.01 & 9850 & 4.12 & 9759 & 4.05 & 200 & 11.37 & 0.04 & 1.06 & 0.09 & 30463 \\
\hline 47827 & -0.016 & 0.145 & 1.009 & 2.860 & 1 & 0.00 & 10160 & 3.94 & 10009 & 3.89 & 30 & 5.31 & 0.12 & -0.32 & 0.26 & 31859 \\
\hline 56341 & -0.007 & 0.141 & 1.087 & 2.867 & 1 & 0.00 & 9780 & 3.77 & 9597 & 3.75 & 30 & 3.58 & 0.19 & -0.89 & 0.41 & 35132 \\
\hline 60629 & -0.014 & 0.169 & 0.966 & 2.906 & 5 & -0.02 & 10070 & 4.42 & 9986 & 4.21 & 50 & 9.65 & 0.07 & 1.56 & 0.16 & 36837 \\
\hline 63112 & 0.008 & 0.117 & 1.089 & 2.831 & 1 & 0.02 & 9990 & 3.55 & & & 10 & 3.39 & 0.22 & -1.04 & 0.47 & 37951 \\
\hline 63584 & -0.014 & 0.135 & 1.013 & 2.870 & 5 & 0.00 & 10200 & 4.01 & 10226 & 4.07 & 20 & 8.09 & 0.06 & 0.70 & 3 & 37720 \\
\hline 67725 & 0.024 & 0.110 & 1.131 & 2.804 & 1 & 0.03 & 9870 & 3.30 & & & 250 & 4.57 & 0.17 & -0.57 & 0.37 & 39898 \\
\hline 69589 & 0.006 & 0.155 & 1.039 & 2.900 & 5 & 0.01 & 9720 & 4.19 & 9481 & 4.05 & 100 & 5.37 & 0.14 & 0.21 & 0.30 & 40561 \\
\hline 71043 & -0.001 & 0.157 & 1.014 & 2.918 & 5 & 0.00 & 9770 & 4.38 & 9575 & 4.23 & 200 & 13.68 & 0.03 & 1.57 & 0.08 & 41081 \\
\hline 71155 & -0.005 & 0.158 & 1.024 & 2.897 & 5 & 0.00 & 9780 & 4.20 & 9768 & 4.10 & 150 & 26.09 & 0.03 & 0.99 & 0.07 & 41307 \\
\hline 71576 & 0.008 & 0.133 & 1.140 & 2.851 & 1 & 0.01 & 9600 & 3.55 & 9234 & 3.69 & 200 & 9.16 & 0.05 & 0.05 & 0.11 & 41003 \\
\hline 74475 & 0.008 & 0.156 & 1.078 & 2.893 & 5 & 0.01 & 9570 & 4.01 & 9336 & 3.99 & 200 & 7.43 & 0.08 & 0.73 & 0.17 & 42775 \\
\hline 76346 & -0.011 & 0.143 & 0.973 & 2.881 & 1 & 0.00 & 10260 & 4.22 & 10190 & 4.14 & 200 & 11.01 & 0.04 & 1.22 & 0.09 & 43620 \\
\hline 79108 & -0.004 & 0.153 & 1.039 & 2.884 & 5 & 0.00 & 9830 & 4.05 & 9767 & 4.14 & 150 & 8.68 & 0.10 & 0.84 & 0.22 & 45167 \\
\hline 80950 & -0.014 & 0.165 & 0.934 & 2.910 & 5 & -0.01 & 10180 & 4.50 & 10175 & 4.20 & 30 & 12.37 & 0.04 & 1.32 & 0.09 & 45585 \\
\hline 84461 & -0.025 & 0.140 & 1.045 & 2.865 & 1 & -0.01 & 10290 & 3.88 & 10075 & 3.85 & 30 & 9.19 & 0.05 & 0.37 & 0.12 & 47717 \\
\hline 85504 & -0.017 & 0.137 & 1.040 & 2.840 & 1 & 0.00 & 10150 & 3.70 & 10259 & 4.01 & $20-30$ & 3.11 & 0.31 & -1.52 & 0.68 & 48414 \\
\hline 87344 & -0.024 & 0.123 & 0.905 & 2.779 & 1 & 0.00 & 10930 & 3.63 & 10725 & 3.71 & 30 & 6.83 & 0.26 & 0.47 & 0.57 & 49321 \\
\hline 87363 & 0.005 & 0.178 & 1.029 & 2.902 & 5 & -0.01 & 9440 & 4.22 & 9306 & 4.20 & 150 & 10.85 & 0.05 & 1.29 & 0.11 & 49259 \\
\hline 87887 & -0.006 & 0.125 & 1.082 & 2.829 & 1 & 0.01 & 9950 & 3.55 & 9913 & 3.73 & 20 & 11.35 & 0.10 & -0.29 & 0.21 & 49641 \\
\hline 92845 & 0.002 & 0.144 & 1.131 & 2.851 & 1 & & 9550 & 3.56 & 9210 & 3.49 & 80 & 5.39 & 0.13 & -0.76 & 0.28 & 52407 \\
\hline 97585 & -0.002 & 0.131 & 1.107 & 2.834 & 1 & 0.01 & 9780 & 3.52 & 9678 & 3.68 & 175 & 6.84 & 0.11 & -0.47 & 0.24 & 54849 \\
\hline 99922 & 0.030 & 0.162 & 1.033 & 2.905 & 5 & 0.02 & 9400 & 4.25 & & & 50 & 8.08 & 0.11 & 0.22 & 0.23 & 56078 \\
\hline 101615 & 0. & 0.169 & 1.00 & 2.920 & 5 & 0.1 & 9580 & 4.41 & 9420 & 4.30 & 10 & 15.58 & 0.04 & 1.50 & 9 & 57013 \\
\hline 104039 & 0.017 & 0.140 & 1.177 & 2.873 & 5 & 0.03 & 9580 & 3.60 & & & $\leq 10$ & 3.66 & 0.19 & -0.90 & 0.42 & 58436 \\
\hline 104430 & -0.006 & 0.148 & 1.005 & 2.895 & 5 & 0.01 & 10040 & 4.24 & 9816 & 4.04 & 50 & 10.56 & 0.07 & 1.23 & 0.14 & 58642 \\
\hline 106797 & 0.007 & 0.16 & 0.90 & 2.88 & 1 & & 9820 & 4.42 & 9674 & 4.18 & 15 & 9.72 & 0.0 & 1.00 & & 59898 \\
\hline 109573 & 0.006 & 0.157 & 0.986 & 2.926 & 5 & 0.0 & 9790 & 4.49 & 9774 & 4.30 & 150 & 14.91 & 0.05 & & 1 & 61498 \\
\hline 111519 & 0.017 & 0.153 & 1.099 & 2.899 & 5 & 0.02 & 9520 & 4.00 & & & 30 & 5.71 & 0.13 & -0.05 & 0.28 & 62655 \\
\hline 111786 & 0.161 & 0.131 & 0.80 & 2.773 & 1 & 0.1 & 7450 & 3.93 & 7397 & 4.29 & $10+150$ & 16.62 & 0.04 & 2.25 & 0.0 & 62788 \\
\hline 113852 & 0.006 & 0.178 & 1.035 & 2.911 & 5 & -0.01 & 9400 & 4.27 & 9249 & 4.11 & 50 & 14.22 & 0.06 & 1.39 & 0.12 & 64003 \\
\hline 114570 & 0.034 & 0.145 & 1.078 & 2.863 & 1 & 0.04 & 9710 & 3.78 & & & 250 & 11.45 & 0.05 & 1.03 & 0.12 & 64466 \\
\hline 125473 & -0.016 & 0.137 & 1.00 & 2.823 & 1 & & 10270 & 3.68 & 9918 & 3.77 & $100-150$ & 13.19 & 0.07 & -0.35 & 0.1 & 70090 \\
\hline 129791 & 0.034 & 0.139 & 0.969 & 2.872 & 5 & 0.04 & 10080 & 4.18 & & & 300 & 7.72 & 0.12 & 1.17 & 0.26 & 72192 \\
\hline 139129 & 0.009 & 0.128 & 1.157 & 2.877 & 1 & 0.01 & 9560 & 3.67 & 9276 & 3.69 & 30 & 7.13 & 0.10 & -0.35 & 0.22 & 76618 \\
\hline 151527 & 0.172 & 0.067 & 1.079 & 2.793 & 6 & 0.05 & 7610 & 3.31 & & & 250 & 7.30 & 0.25 & -0.13 & 0.53 & 82259 \\
\hline & & & & & 1 & 0.19 & 10130 & 3.47 & & & & & & 0.47 & 0.53 & \\
\hline 152849 & -0.005 & 0.141 & 0.995 & 2.864 & 1 & 0.01 & 10210 & 4.02 & 10030 & 4.07 & 200 & 8.99 & 0.15 & 0.29 & 0.32 & 82925 \\
\hline 160263 & & 0.116 & 1.12 & 2.8 & 1 & & & 3.48 & & & 75 & 4.94 & 0.18 & -0.83 & 0.40 & 86552 \\
\hline 170680 & 0.006 & 0.140 & 1.052 & 2.892 & 5 & 0.02 & 9980 & 4.09 & & & 200 & 15.30 & 0.05 & 0.96 & 0.12 & 90806 \\
\hline 188228 & -0.008 & 0.145 & 0.959 & 2.897 & 5 & & 10230 & 4.38 & 10146 & 4.28 & 100 & 30.73 & 0.02 & 1.36 & 0.04 & 98495 \\
\hline 193571 & -0.007 & 0.170 & 0.994 & 2.915 & 5 & -0.01 & 9820 & 4.41 & 9652 & 4.25 & 80 & 13.35 & 0.06 & 1.22 & 0.13 & 100469 \\
\hline 213320 & -0.017 & 0.140 & 1.015 & 2.863 & 1 & 0.00 & 10200 & 3.95 & 10351 & 4.05 & $20-30$ & 12.29 & 0.11 & 0.29 & 0.23 & 111123 \\
\hline 213398 & -0.004 & 0.167 & 1.045 & 2.910 & 5 & -0.01 & 9640 & 4.24 & 9487 & 4.15 & 30 & 21.99 & 0.04 & 1.00 & 0.08 & 111188 \\
\hline & & & & & & & & & 7558 & 4.06 & 50 & 11.76 & 0.07 & 1.95 & 0.14 & 113331 \\
\hline 218639 & 0.005 & 0.152 & 1.037 & 2.884 & 5 & 0.01 & 9790 & 4.06 & 9391 & 4.06 & 200 & 9.45 & 0.11 & 1.26 & 0.23 & 114371 \\
\hline 223352 & -0.004 & 0.154 & 1.019 & 2.893 & 5 & 0.00 & 9860 & 4.19 & 9706 & 4.14 & 300 & 22.73 & 0.05 & 1.37 & 0.11 & 117452 \\
\hline 225200 & 0.011 & 0.133 & 1.097 & 2.825 & 1 & 0.02 & 9780 & 3.51 & & & 300 & 7.75 & 0.12 & 0.74 & 0.26 & 345 \\
\hline
\end{tabular}


When the value of the colour excess has been found to be negative, no correction has been applied to the colour indices $T_{\text {eff }}$ and $\log g$.

\section{3. $T_{\mathrm{eff}}$ and $\log g$ from Geneva photometry}

We computed the atmospheric parameters from the Geneva photometric indices by using the recent calibration by Künzli et al. (1997).

This system provides an independent way to determine $T_{\text {eff }}$ and $\log g$ since it does not include any filter centered on a Balmer line. We limited this computation to undereddened stars, i.e. to those which, according to uvby $\beta$ photometry, have a colour excess $E(b-y)$ in the range \pm 0.01 . The computed $T_{\text {eff }}$ and $\log g$ are given in Table 2, Cols. 10 and 11 .

\subsection{Comparison of $T_{\text {eff }}$ and $\log g$ values}

The atmospheric parameters derived from the two photometric system are directly comparable only for stars with $E(b-y)=0.0$. In order to have a larger sample, we consider as unreddened the stars with $E(b-y) \leq 0.01$ and, in order to have homogeneous data, the MD parameters have been recalculated for this comparison without taking into account the colour excess for stars with $E(b-y)=0.01$.

Figures 1a and $1 \mathrm{~b}$ display the results for $T_{\text {eff }}$ and $\log g$ respectively; Fig. 1a does not include the abnormally low values of $T_{\text {eff }}$ derived from both photometric systems for the $\lambda$ Boo star HD 111786, which is, in fact, a binary, as demonstrated by Faraggiana et al. (1997).

We check the consistency of the two sets of $T_{\text {eff }}$ and $\log g$ and we look for possible systematic differences. The values of $T_{\text {eff }}$ from the $u v b y \beta$ are systematically higher than those from the Geneva photometry. This difference is not related to the stellar rotation as it appears from the absence of any systematic relation between the $\Delta T_{\text {eff }}$ and the $v \sin i$ value. Nevertheless, the difference between these two sets of values is small, the average being $150 \mathrm{~K}$. The $\log g$ comparison shows higher scatter than that of $T_{\text {eff }}$; the largest difference refers, to the already cited HD 111786. The star HD 85504, is an intriguing object with peculiar spectrum and kinematics (see the Appendix).

For most of the stars, $\log g$ (MD) is higher than $\log g$ (Gen) for large $\log g$ values, while the opposite is true for $\log g$ lower than 4. The correction of $\log g$ (MD) proposed by Napiwotzki et al. (1993), being independent of the $\log g$ value, does not solve this discrepancy.

A discrepancy between $\log g$ values obtained from these two sets of photometric indices, such as for HD 85504 and HD 111786 can be interpreted as a sign of difference in their flux distribution compared to that used
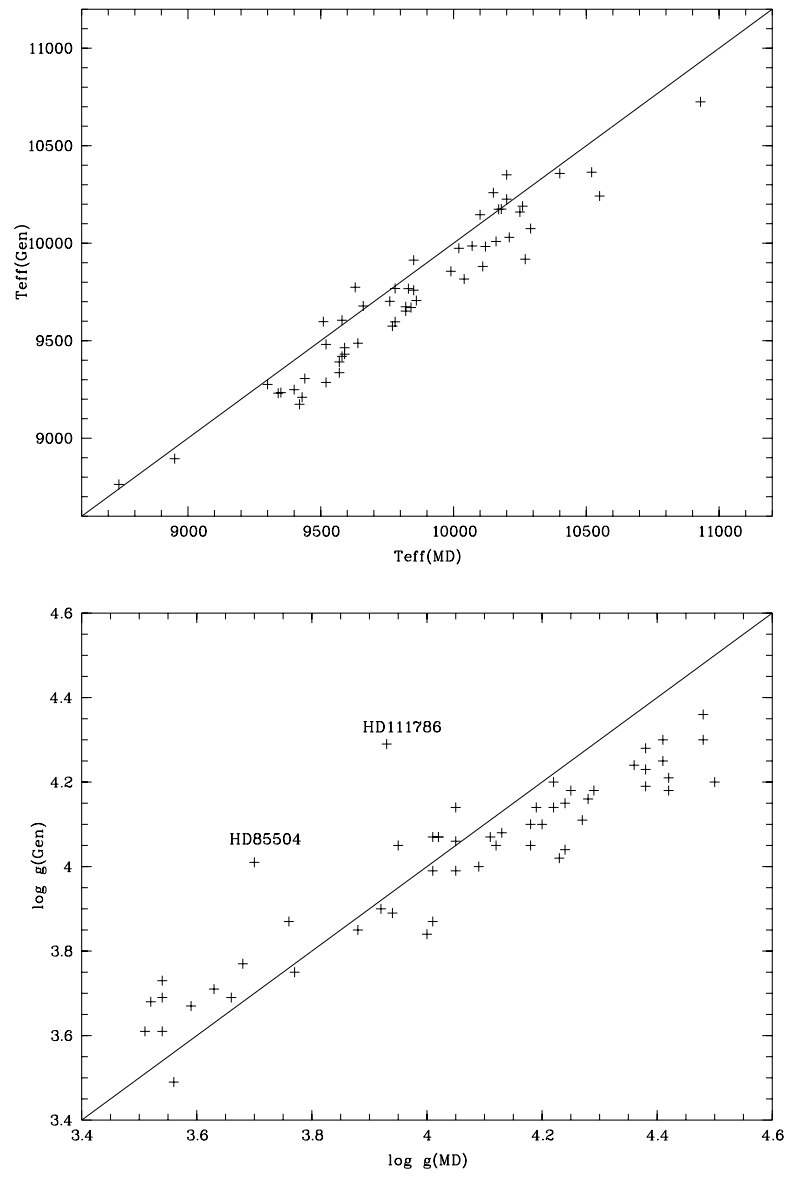

Fig. 1. Relation between $T_{\text {eff }}$ (Fig. 1a) and $\log g$ (Fig. 1b) derived from MD and Geneva calibrations; no dereddening correction are applied to the colours in both systems, and only stars with $E(b-y) \leq 0.01$ are considered

for calibration. The $\mathrm{H}_{\beta}$ intensity plays an important role in the calibration of the Strömgren photometry, so we suggest that for these two stars, such a difference shows that their $\mathrm{H}_{\beta}$ intensity is not coherent with their continuum character.

Independently of the adopted calibration, it is clear that the A0 dwarf stars occupy a broad domain of $T_{\text {eff }}$ and $\log g$ which leads to a loose correlation with spectral type and luminosity class appears. We remind that the internal accuracy reached by different MK classifiers is of \pm 0.7 in luminosity class (Jaschek \& Valbousquet 1997); the $\log g$ parameter plotted in Fig. 1b, cannot be directly related to this luminosity class.

If we exclude the binary HD 111786, the $\lambda$ Boo star HD $31295\left(T_{\text {eff }}(\mathrm{MD})=8763 \mathrm{~K}\right)$ and the peculiarly reddened star HD $151527\left(T_{\text {eff }}(\mathrm{MD})=7610 \mathrm{~K}\right), T_{\mathrm{eff}}(\mathrm{MD})$ spans from $9070 \mathrm{~K}$ (HD 21473) to $10930 \mathrm{~K}$ (HD 87344) and $\log g(\mathrm{MD})$, when only A0 V stars are considered, covers the range from 3.30 (HD 67725) to 4.50 (HD 80950). 


\section{Spectral analysis}

The goal of this study is to establish an HR diagram for nearby A0 stars free of any peculiar object. The comparison between the observed spectra and those computed with the above derived parameters should allow to select the "normal" stars.

A grid of synthetic spectra, covering the range of the previously determined $T_{\text {eff }}$ and $\log g$ values and based on Kurucz stellar model atmosphere (Kurucz 1993) has been constructed; solar abundances and a microturbulence value equal to $2 \mathrm{~km} \mathrm{~s}^{-1}$ are adopted. Computations have been made with various rotational broadening values for each synthetic spectrum.

From the grid of the Kurucz fluxes (1993) we computed also the UV fluxes in the four TD1 bands; the comparison between the observed and computed colour indices $m_{\mathrm{UV}-V}$ has been systematically done. The choice of these data is due to the fact that the Thompson et al. (1978) Catalogue contains all but 2 stars of our sample, while only 14 of them have been observed by IUE in the low resolution mode and have spectra available from the Final Archive.

In order to compare the optically observed spectra with the computed ones, the needed shift in wavelength to be applied to the observed spectrum is obtained by cross correlation. The templates used are the spectra computed with the appropriate stellar parameters. The cross correlation program differs from the Midas command XCORRELATE in the sense that the correlation index is normalized to 1 . The template and the spectrum are rebinned in the velocity space, the rebinning being largely oversampled, the shift is applied and the spectrum rebinned back in wavelength at its original stepsize.

As by-product of this program, the correlation curve contains informations on the quality of the fit between observed and computed spectra.

The broadening parameter (Table 2, Col. 14) refers to the $v \sin i$ value adopted for the best fit of the observed and computed spectrum and, in the case of spectroscopic binaries, does not have any physical meaning.

The selection of the normal stars follows from the application of tests based on:

\section{- The correlation curve}

The height and the shape of the correlation curve are related to similarity of the synthetic spectrum and the observation; in particular, a non symmetric correlation curve indicates a possible binarity.

\section{- Coherence between MD and Geneva parameters}

The comparison between the MD and Geneva parameters for stars with $E(b-y)=0.00$ shows that the largest difference in $T_{\text {eff }}$ is $352 \mathrm{~K}$ (HD 125473), significantly larger than the uncertainties expected for the $T_{\text {eff }}$ determination from errors on photometric colour indices. We note that for this star the MD parameters are those from which the best fit with the computed spectrum is obtained.

We remark that for several stars the $\log g$ computed by MD from Strömgren photometry is higher than what expected for dwarf A0 stars. We looked at the eleven stars with $\log g$ higher than 4.3 (HD 3003, HD 16152, HD 38206, HD 60629, HD 71043, HD 80950, HD 101615, HD 106797, HD 109573, HD 188228, HD 193571). None of them has an $\mathrm{H}_{\gamma}$ profile which fits the spectrum computed with the MD parameters. For all these stars $E(b-y) \leq 0.01$, so that the Geneva parameters are computed as well. The $\log g$ computed from the Geneva photometry is systematically lower and the spectrum computed with Geneva parameters from undereddened colours, fits better the $\mathrm{H}_{\gamma}$ profile.

\section{- Observed and computed profiles}

The core of the observed $\mathrm{H}_{\gamma}$ is expected to be deeper than that computed for the appropriate $v \sin i$ value with Kurucz models which do not include NLTE effects; therefore an observed profile shallower than the computed one is a sign of abnormality. In highly rotating stars $\mathrm{Mg}$ II 4481 is the only metallic feature with a measurable profile; this feature is expected to be rotationally broadened, as soon as the $0.02 \AA$ separation of the Mg II doublet becomes irrelevant compared to the stellar $v \sin i$ value.

\section{Spectroscopic behaviour of double stars}

According to the values given Table 1 Cols. 5 and 6 we can suspect that the photometric indices and consequently the derived atmospheric parameters, are affected by a companion for 10 stars. The $\Delta m$ and the angular separation is such that the spectra of five of these binaries must be composite: HD 3003, HD 4065, HD 47827, HD 104039, HD 152849.

According to our analysis of the spectra we note:

- HD 3003: The correlation curve is not symmetric and the Mg II 4481 is not rotationally broadened (Figs. 2a and $\mathrm{b}$ ).

- HD 4065: The profile of $\mathrm{H}_{\gamma}$ is not fitted by the computed ones with MD or Geneva parameters and the core of the line is considerably shallower than the computed ones (Figs. 3a and b); the Mg II 4481 has a very peculiar profile and the metal lines are poorly reproduced by computations (Fig. 3c).

- HD 47827: The Mg II profile has broad wings as in the previous star; $\mathrm{H}_{\gamma}$ is slightly asymmetric and flatter than the computed one and the metallic lines are too weak for a non peculiar star.

- HD 104039: The duplicity is dubious according to the Remarks in the BSC. This star has $E(b-y)=0.03$ 

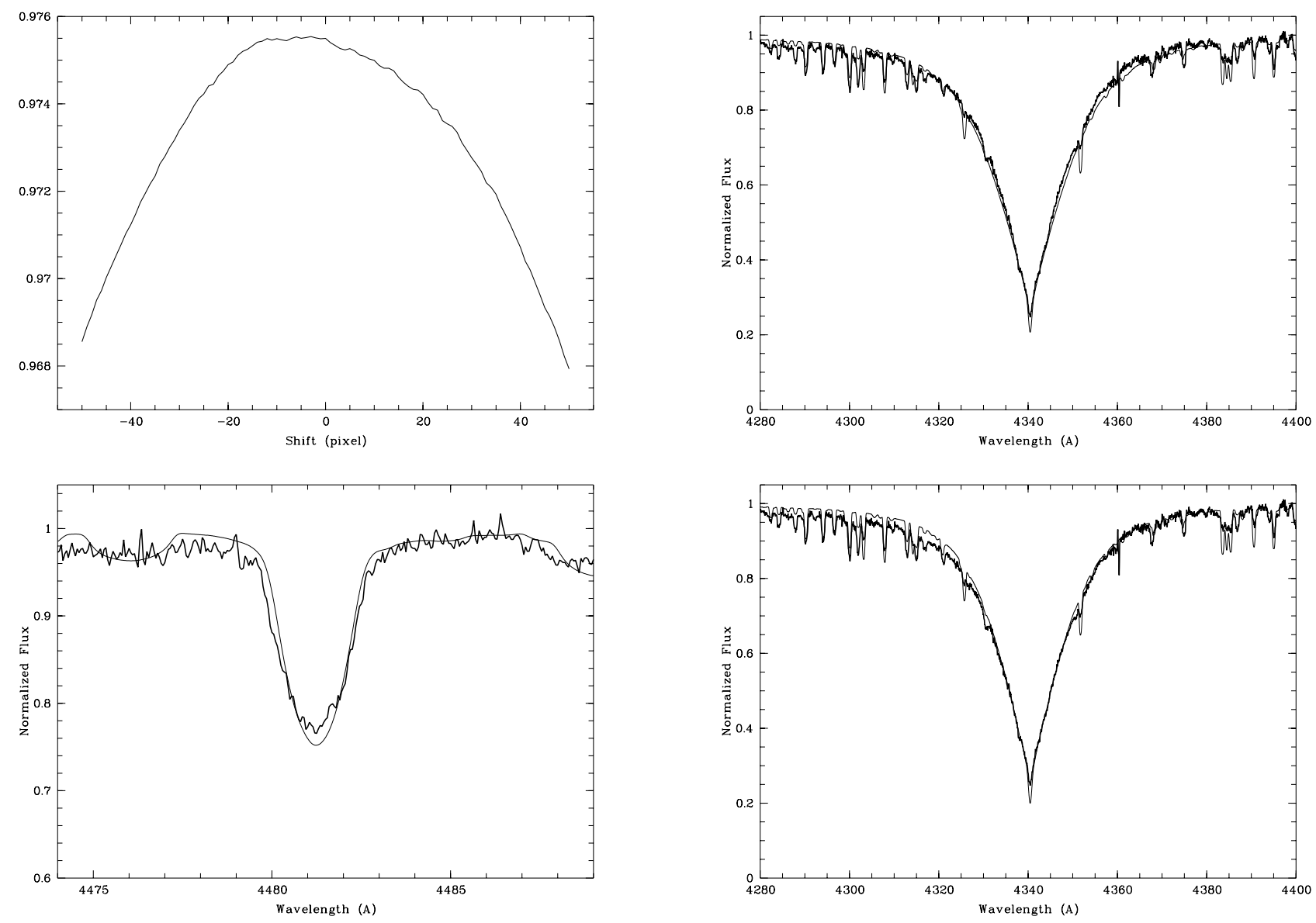

Fig. 2. a) the cross correlation between the observed spectrum of HD 3003 and the computed one $(1$ pixel $=0.05 \AA)$. b) the $\mathrm{Mg}$ II 4481 line (thick line) overplotted on the computed one (thin line)

as HD 7916 (see below). $\mathrm{H}_{\gamma}$ does not fit the synthetic spectrum computed with the MD parameters taking into account the reddening; the metal lines are stronger than the computed ones. A better fit is obtained with the spectrum computed with the parameters corresponding to no colour excess, but the metal lines intensities do not agree even with these parameters; also the TD1 colour indices fit better those computed with no colour excess.

- HD 152849: The spectrum is of low $\mathrm{S} / \mathrm{N}$ quality and the line profiles cannot be as well defined as in most of the other spectra but the profile of $\mathrm{Mg}$ II 4481 is non rotationally broadened (Fig. 4) and we have checked that this conclusion does not rely upon the position of the continuum.

We conclude that, at the resolution of our spectra, the contamination by a companion star can be detected by at least one of the criteria we have chosen:

a) non symmetry of the cross correlation curve (see Fig. 2a)

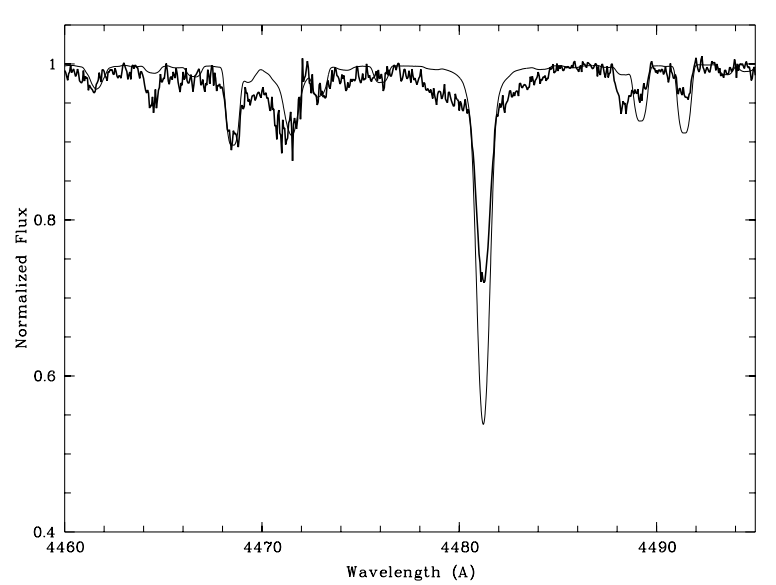

Fig. 3. a) the observed $\mathrm{H}_{\gamma}$ (thick line) of HD 4065 overplotted on that computed with MD parameters. b) the same, but with Geneva parameters. c) the Mg II 4481 region compared with the computed spectrum (at $\lambda 4470$ a defect of the CCD is present) 


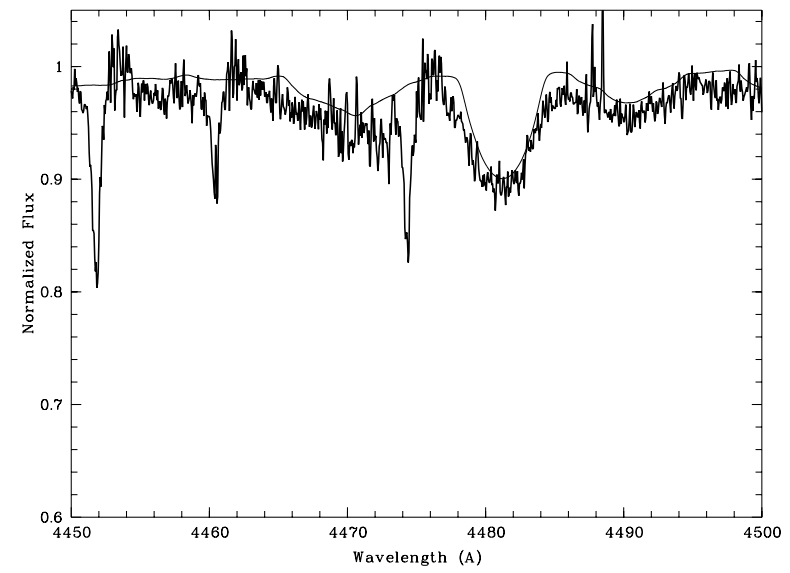

Fig. 4. The Mg II 4481 region of HD 152849 (thick line) compared with the computed spectrum (thin line); the narrow features at $\lambda \lambda 4452,4460$ and 4473 correspond to defects of the CCD $3 \mathrm{c}, 4)$

b) peculiar profile of $\mathrm{Mg}$ II 4481 line (see Figs. 2b,

c) distorted line profiles and/or flat $\mathrm{H}_{\gamma}$ core (see Fig. 3a).

In this temperature range the $\mathrm{H}_{\gamma}$ line is so strong that the cross correlation is dominated by its profile; if the combined profile of a binary star can be reproduced by a model, the duplicity detection is confined to the behaviour of the metal lines.

For the other 5 binaries, HD 7916, HD 17864, HD 34968, HD 99922, HD 213320, the $\Delta m$ and/or their separation are such that, a priori, their spectra will not be affected by the companion. Nevertheless, we found some peculiarities in their spectra.

- For HD 7916 the Mg II 4481 is broader than the other metal lines and we found indications of duplicity in the line profiles; the profiles of $\mathrm{H}_{\gamma}$ and $\mathrm{Mg}$ II 4481 (Figs. 5a and b) indicate the presence of a secondary companion on the short wavelength side and these peculiarities cannot be due to the visual companion which has a too faint magnitude according to the $\Delta m$ given in Table 1 ; however a lower magnitude difference, $\Delta V=1.95$ has been measured by the Hipparcos experiment in good agreement with the value (1.84), estimated by Corbally (1984). We note that HD 7916 is one of the most reddened stars; we interpret this colour excess as an ad hoc quantity needed to reproduce the colours of a single normal star.

- For HD 17864 The Mg II profile is peculiar: flat and its broadening does not correspond to a rotational broadening. The $\mathrm{H}_{\gamma}$ profile is asymmetric with a too flat core.

- For HD 99922 the magnitude difference and the separation are too large to affect our spectroscopic data; unfortunately, the $\mathrm{Mg}$ II 4481 region is not available on our spectrum. Neverthless we note that the other metal lines are abnormally shallow and weak and strongly suggest a
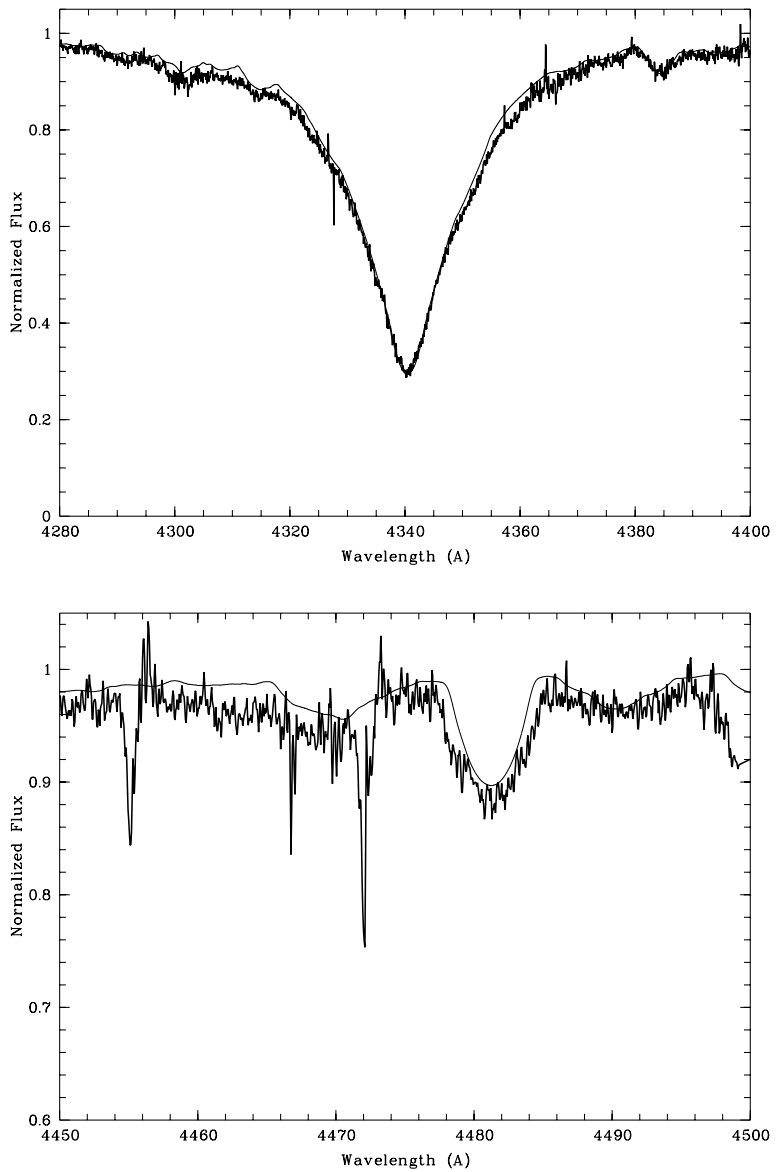

Fig. 5. The observed and computed spectra of HD 7916 in the regions of $\mathrm{H}_{\gamma}$ (Fig. 5a) and Mg II 4481 (Fig. 5b); sharp and narrow absorptions are due to bad CCD columns

"veiling" effect (Fig. 6), according to the definition introduced by Corbally (1987). We interpret these peculiarities as due to a source which cannot be identified with the known companion.

- In the spectrum of HD 34968 only a slight asymmetry of the MgII line profile is detected.

- For HD 213320 the $\mathrm{H}_{\gamma}$ profile is very well reproduced by computations; in spite of this, the metallic lines present distorted profiles and their intensity is poorly reproduced by the synthetic spectrum.

So we note that unexpectedly distorted line profiles are found also among these 5 stars.

\section{Search for reference stars}

Using the criteria listed above, we extend a similar analysis to all the sample stars. The results are summarized in Table 3 . 


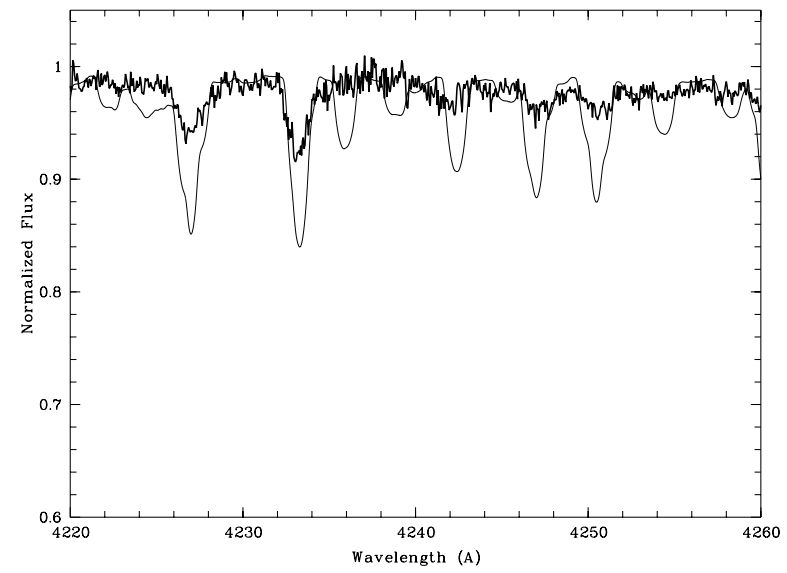

Fig. 6. The observed (thick line) and the computed (thin line) spectra of HD 99922 shown a pronounced "veiling" effect on the metal lines

From this analysis it appears that a large percentage of our sample stars (being either binaries or peculiar) cannot be used to build a set of reference stars.

\subsection{Newly detected binaries}

For 13 stars the cross correlation curve has a highly distorted shape (see, for example, the cross correlations of HD 36473 and HD 56341 in Figs. 7a and b). In all the spectra of these stars the line profiles are distorted; details on each star are summarized in Table 3 and in the Appendix.

These stars are:

HD 16152, HD 21473, HD 22789, HD 30397, HD 36473, HD 56341, HD 104430, HD 106797, HD 111519, HD 111786, HD 113852, HD 139129, HD 151527.

HD 111786 has been discussed by Faraggiana et al. (1997) and its highly distorted UV colours are plotted in Fig. 8b.

HD 139129 is the only star for which spectroscopic informations are found in the literature (see Appendix); the peculiar abundances, derived by Lemke $(1989,1990)$, strengthen our binary interpretation.

HD 151527 is an abnormally reddened star; the choice of the Strömgren group according to the lowest value of the colour excess produces atmospheric parameters in conflict with observations; from $E(b-y)=0.19$ the derived atmospheric parameters produce a synthetic spectrum which agrees with observed $\mathrm{H}_{\gamma}$, but not with the metal lines spectrum. We note also the low value of the derived $\log g$ value, in disagreement with its MK classification. The distorted UV colours of this star are plotted Fig. 8c; this star and HD 111786 are the only two objects for which a large discrepancy between observed and computed UV colours is found.
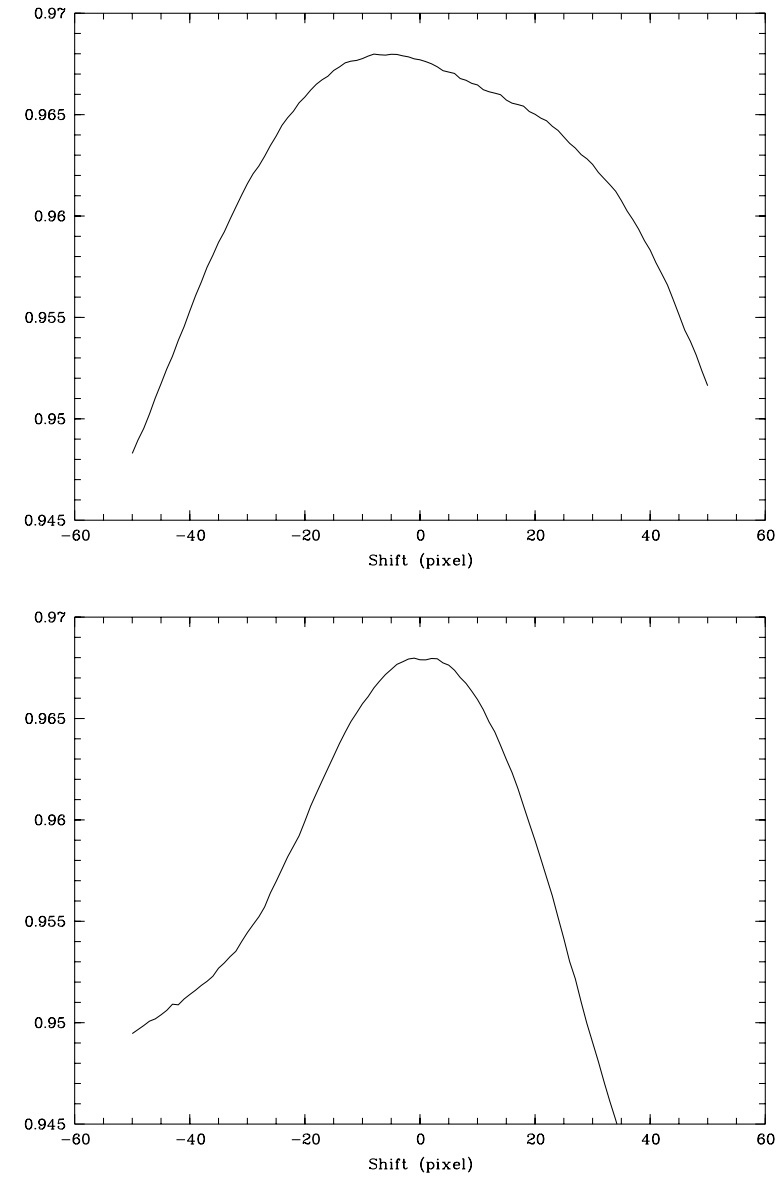

Fig. 7. The distorted cross correlations of HD 36473 a) and of HD $56341 \mathbf{b}$ ) indicate the presence of a secondary shifted source. (1 pixel = $0.05 \AA$ )

According to the Hipparcos and Tycho Catalogues (ESA, 1997) HD 111519, HD 113852 and HD 151527 are probable astrometric binaries, due to the flag in field 59 of the Hipparcos Catalogue.

\subsection{Suspected binaries}

For other stars, even if the cross correlation is not as distorted as for the previous ones, the spectral peculiarities and the distorted line profiles suggest a binary nature of the observed objects or some peculiarity.

The 9 stars suspected to be binaries on the basis of the visual and UV data are: HD 15646, HD 20980, HD 71043, HD 79108 HD 84461, HD 114570, HD 129791 HD 188228 and HD 193571. Details on them can be found in Table 3 and in the Appendix.

We note that HD 188228 belongs to the system of standard stars for rotational velocity determinations defined by Slettebak et al. (1975); the $v \sin i$ value derived by Levato (1972) differs considerably from that by Slettebak et al. 
Table 3. Summary of the spectral analysis

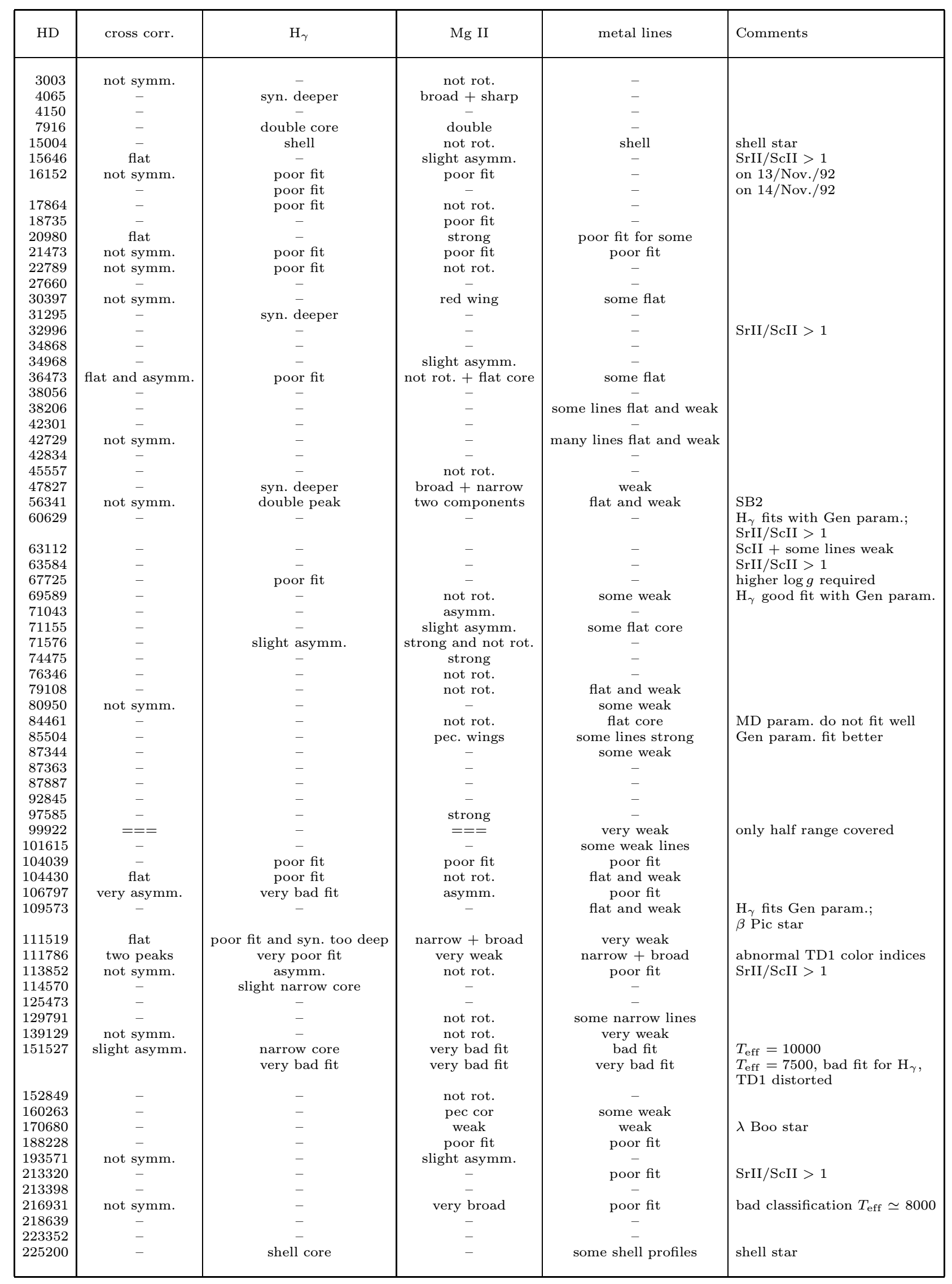



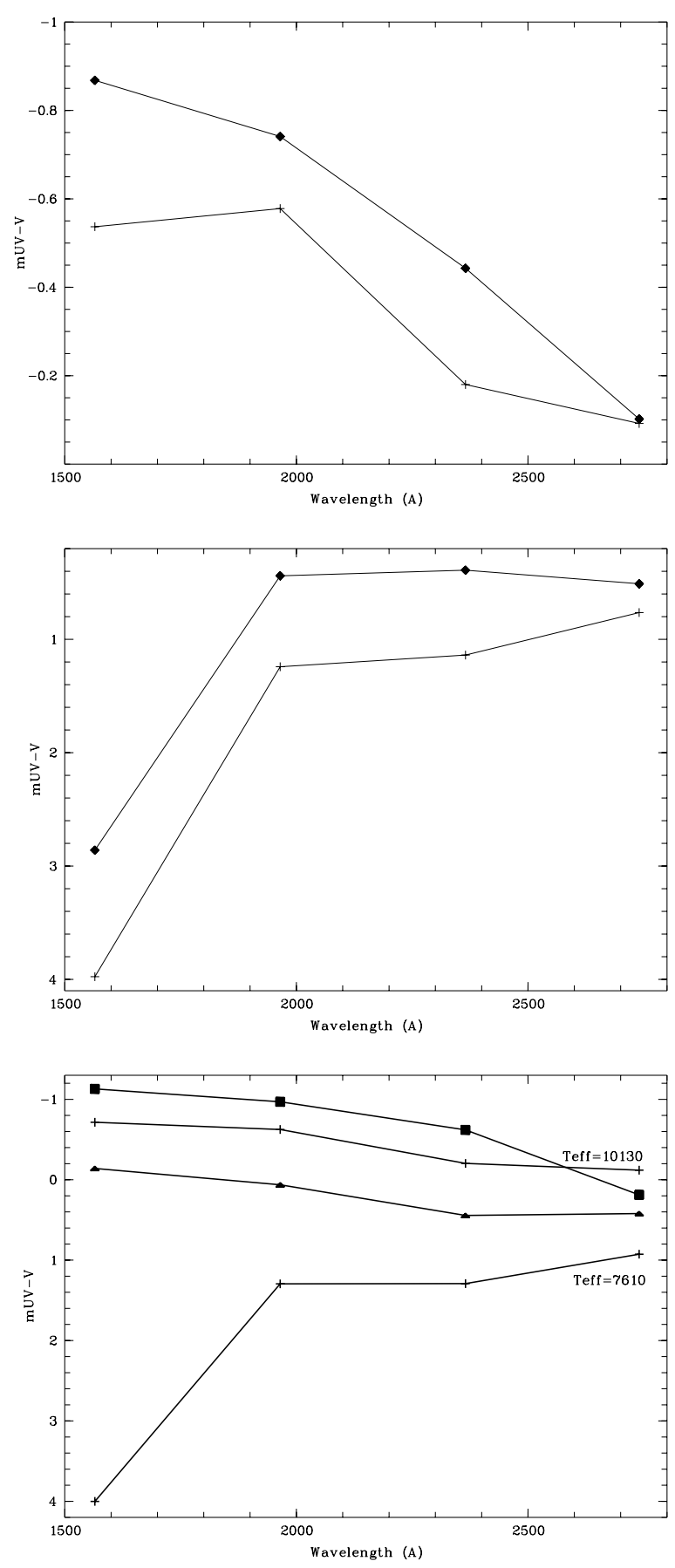

Fig. 8. The observed $U V$ colour indices $m_{\mathrm{UV}}-V$ dereddened according to the $E(b-y)$ given in Table 2 and compared with those computed with the MD atmospheric parameters for a) HD 109573, b) HD 111786 and c) HD 151527 where the filled triangle refer to $E(b-y)=0.05$ and filled square to $E(b-y)=0.19$. The computed values are indicated with $(+)$. (see comments on HD 109573 in the Appendix)
For HD 129791, in the Hipparcos Catalogue (ESA, 1997), a note in the field 59 indicates that it is probably an astrometric binary.

\subsection{Remarks from Hipparcos astrometric data}

In the Hipparcos Catalogue (ESA, 1997) besides the stars for which a companion is observed and its motion described, there are several stars from Table 1 considered as probable astrometric binaries on the basis of their astrometric solution.

They are: HD 4150, HD 42301, HD 63584, HD 87887 , HD 92845, HD 109573 HD 111519, HD 113852, HD 129791, HD 151527, HD 223352.

Among these 11 stars only 4 present spectral peculiarities indicating a possible binarity. We cannot expect to have a one to one coincidence because the detection of binarity indices in the spectra at our disposal requires a small difference in magnitude and separation, and a difference in radial velocity if the two components have similar rotational velocities.

The other peculiar objects are:

\subsection{Shell stars}

HD 225200 is a shell star; its spectrum is similar to that of the already known shell star HD 15004. The hypothesis of binarity for HD 225200, as suggested by AM, must be rejected because the sharp components are only present on the lines expected in shell stars: the core of $\mathrm{H}_{\gamma}$, all the strong lines of the Fe II Multiplet 27 and the Ti II 4468.

\subsection{A misclassified star}

HD 216931 has been misclassified as A0, according to our two spectra; these two spectra refer to an object much cooler than an A0 star. The parameters given in Table 2 have been derived from the Geneva photometry, neglecting any colour excess, in spite of the $E(B-V)=0.06$. The whole spectrum (and in particular the $\mathrm{H}_{\gamma}$ profile) is not reproduced by that computed with the above parameters. The $\mathrm{H}_{\gamma}$ profile is fitted by a spectrum computed with a temperature $500 \mathrm{~K}$ higher than the predicted one, (so that the neglected reddening can be at the origin) i.e. by the spectrum computed with $T_{\text {eff }}=8000 \mathrm{~K}$ and $\log g=4.0$, values which produce a fairly good agreement also between observed and computed UV colours. However several metal lines are poorly fitted by the synthetic spectrum computed with these parameters; the ratio SrII4215/ScII4247 of the observed spectrum is much higher than the computed one, as in Am stars; several stronger than computed features are present on the two 
observed spectra. The Mg II 4481 is distorted and very broad compared to other metal lines.

The presence of a companion which cannot be identified with the known $\mathrm{B}$ and $\mathrm{C}$ components of this visual triple system (ADS 16392) is reported by Richichi et al. (1997) from lunar occultations observed in the near infrared and must be at the origin of the observed peculiarities and of the discrepancy between our two spectra and the MK classification.

\section{Results of the search for reference stars}

\subsection{Remarks on cross correlation}

The cross correlation shape is highly dominated by the strong $\mathrm{H}_{\gamma}$ line which cannot be recorded, at high resolution, on a single order of an echelle spectrograph. Even if great care has been taken in connecting the orders and on the normalization procedures, a doubt remains on the reliability of $\mathrm{H}_{\gamma}$ profile, since the continum between the very broad wings has been placed by connecting them with a straight line.

The possibility that instrumental effects are not totally eliminated by the Flat Field correction cannot be discarded, the light path not being the same for the star and for the tungsten lamp. Consequently, it remains a possible source of some of the slight asymmetries of $\mathrm{H}_{\gamma}$ profiles and of the cross correlation shape which is dominated by the effect of this strong line profile.

\subsection{Remarks on colour excess}

There are 7 stars (HD 7916, HD 67725, HD 104039, HD 114570, HD 129791, HD 151527, HD 216931) for which $E(b-y)$ is higher than 0.02 . For all, but HD 67725, spectral peculiarities have been detected (see previous section); for HD 67725 we note that the $\log g$ value derived from the colours is abnormally low for an A0 V star and the corresponding computed $\mathrm{H}_{\gamma}$ does not fit the observed one.

So all the reddened stars present some spectral peculiarity.

\subsection{Remarks on hot Am detection}

Among the CP stars the strong lines of Si II and Mn II lie out of our observed spectral range so preventing us to detect hot Ap stars.

We can detect the Am character through one of the classical criteria, the SrII4215/ScII4247 ratio higher than that expected for solar abundances. According to this criterium, the mild hot Am candidates are: HD 32996, HD 63584 and 213320.
We note also that for two suspected binaries, (see Table 3), HD 15646 and HD 113852, the ratio SrII $4215 / \mathrm{ScII} 4247$ is much higher than that expected for solar abundances. A spectral classification based only on this ratio may wrongly assign to the Am class an object that is, in reality, a spectroscopic binary whose components do not differ much in luminosity. This may be the case for HD 60629; several metal lines show an abnormal core.

\subsection{Reference stars}

We conclude that from our original sample of 71 stars at least 21 (the 8 out of the 10 stars discussed in Sect. 5 (i.e. all, but HD 34968 and HD 213320) and the 13 stars discussed in Sect. 6.1) cannot be considered as reference stars, so that the masses and ages are computed for a reduced sample of 50 stars only.

However, doubts remain on other stars, due to possible binarity as indicated in Sect. 6.2; for several of the remaining stars the peculiarities are not strong enough to exclude any instrumental origin; however, a significant reddening remains difficult to accept for such nearby stars, large discrepancies between results obtained by other authors on $v \sin i$ (e.g. the discrepant results found for HD 4150) and more subtle discrepancies as those discussed in the Appendix, suggest that the number of binaries and/or peculiar objects may be higher than that selected in this section.

High rotation is expected to affect the photometric colours (Collins \& Smith 1985) and the line intensities (Slettebak et al. 1980) of A-type stars. The weakening of Hgamma and Mg II 4481 lines due to rotation is computed in the latter paper; we could not reveal any correlation between the intensities of these lines and the $v \sin i$ values, the rotational effect being probably masked either by the unknown $i$ value and/or by the fact that most of the non binary stars of our sample have $v \sin i$ lower than $200 \mathrm{~km} \mathrm{~s}^{-1}$. Also a relation between the spread on the HR diagram (see next section) and the stellar vsini has been searched, but not detected. In particular no trend between $\log g$ and $v \sin i$ appears, nor are the oldest stars those with lower $v \sin i$.

\section{Mass and age of dwarf A0 stars}

\subsection{The HR diagram}

Hipparcos parallaxes enable us to establish the HR diagram for this set of stars, in order to determine their masses and ages.

The effective temperature is that derived from photometric calibrations. The visual absolute magnitudes 
Table 4. Parameters computed from the HR diagram and evolutionary tracks for the reduced sample; the star HD 216931 has been excluded from this table as well as HD 27660. The evolutionary tracks used for these computations are those by Schaller et al. (1990). The errors are given for the determination of $\log (L / L \odot), \mathcal{M} / \mathcal{M}_{\odot}, \log g_{\mathrm{ev}}$. For the ages the error is given in percentage

\begin{tabular}{|c|c|c|c|c|c|c|c|c|c|c|}
\hline HD & $\log \left(L / L_{\odot}\right)$ & $\sigma$ & $\mathcal{M} / \mathcal{M}_{\odot}$ & $\sigma$ & $R / R_{\odot}$ & $\log g_{\mathrm{ev}}$ & $\sigma$ & Age $(\log (\mathrm{yrs}))$ & $\sigma(\%)$ & HIP \\
\hline 4150 & 2.01 & 0.04 & 2.82 & 0.07 & 3.52 & 3.80 & 0.03 & 8.51 & 7 & 3405 \\
\hline 15004 & 2.11 & 0.18 & 2.98 & 0.24 & 3.64 & 3.79 & 0.14 & 8.45 & 11 & 11261 \\
\hline 15646 & 1.60 & 0.06 & 2.40 & 0.08 & 2.11 & 4.17 & 0.05 & 8.42 & 34 & 11479 \\
\hline 18735 & 1.65 & 0.07 & 2.39 & 0.09 & 2.43 & 4.05 & 0.06 & 8.57 & 17 & 13947 \\
\hline 20980 & 1.71 & 0.09 & 2.45 & 0.10 & 2.59 & 4.00 & 0.07 & 8.58 & 13 & 15700 \\
\hline 31295 & 1.22 & 0.03 & 1.97 & 0.07 & 1.69 & 4.28 & 0.03 & 8.42 & 70 & 22845 \\
\hline 32996 & 1.72 & 0.07 & 2.56 & 0.09 & 2.19 & 4.17 & 0.06 & 8.34 & 37 & 23777 \\
\hline 34868 & 1.89 & 0.07 & 2.70 & 0.10 & 2.88 & 3.95 & 0.06 & 8.49 & 11 & 24831 \\
\hline 34968 & 2.44 & 0.08 & 3.47 & 0.15 & 5.27 & 3.54 & 0.07 & 8.35 & 10 & 24927 \\
\hline 38056 & 1.74 & 0.06 & 2.57 & 0.09 & 2.29 & 4.13 & 0.05 & 8.40 & 27 & 26796 \\
\hline 38206 & 1.45 & 0.05 & 2.29 & 0.10 & 1.72 & 4.33 & 0.03 & 6.95 & 157 & 26966 \\
\hline 42301 & 1.59 & 0.05 & 2.38 & 0.08 & 2.09 & 4.18 & 0.04 & 8.42 & 34 & 29150 \\
\hline 42834 & 2.09 & 0.09 & 2.93 & 0.13 & 3.84 & 3.74 & 0.07 & 8.48 & 8 & 29304 \\
\hline 42729 & 2.19 & 0.11 & 3.05 & 0.16 & 4.34 & 3.65 & 0.09 & 8.46 & 13 & 29347 \\
\hline 45557 & 1.57 & 0.04 & 2.34 & 0.07 & 2.09 & 4.17 & 0.04 & 8.45 & 33 & 30463 \\
\hline 60629 & 1.43 & 0.06 & 2.26 & 0.10 & 1.71 & 4.33 & 0.04 & 6.97 & 162 & 36837 \\
\hline 63584 & 1.74 & 0.05 & 2.54 & 0.08 & 2.37 & 4.09 & 0.05 & 8.45 & 22 & 37720 \\
\hline 63112 & 2.42 & 0.19 & 3.25 & 0.25 & 5.41 & 3.49 & 0.15 & 8.42 & 15 & 37951 \\
\hline 67725 & 2.22 & 0.15 & 3.11 & 0.20 & 4.41 & 3.64 & 0.12 & 8.44 & 14 & 39898 \\
\hline 69589 & 1.90 & 0.12 & 2.67 & 0.15 & 3.14 & 3.87 & 0.10 & 8.54 & 10 & 40561 \\
\hline 71576 & 1.95 & 0.05 & 2.73 & 0.08 & 3.43 & 3.81 & 0.04 & 8.54 & 8 & 41003 \\
\hline 71043 & 1.36 & 0.03 & 2.17 & 0.09 & 1.68 & 4.33 & 0.03 & 7.05 & 120 & 41081 \\
\hline 71155 & 1.59 & 0.03 & 2.36 & 0.06 & 2.18 & 4.14 & 0.03 & 8.49 & 24 & 41307 \\
\hline 74475 & 1.68 & 0.07 & 2.42 & 0.09 & 2.51 & 4.02 & 0.06 & 8.58 & 15 & 42775 \\
\hline 76346 & 1.53 & 0.04 & 2.37 & 0.08 & 1.86 & 4.28 & 0.01 & 8.14 & 71 & 43620 \\
\hline 79108 & 1.65 & 0.09 & 2.42 & 0.10 & 2.32 & 4.09 & 0.07 & 8.51 & 24 & 45167 \\
\hline 80950 & 1.49 & 0.04 & 2.32 & 0.08 & 1.78 & 4.30 & 0.03 & 7.90 & 91 & 45585 \\
\hline 84461 & 1.88 & 0.05 & 2.70 & 0.08 & 2.74 & 3.99 & 0.04 & 8.47 & 12 & 47717 \\
\hline 85504 & 2.62 & 0.27 & 3.62 & 0.46 & 6.63 & 3.35 & 0.20 & 8.31 & 26 & 48414 \\
\hline 87363 & 1.44 & 0.05 & 2.19 & 0.07 & 1.98 & 4.19 & 0.04 & 8.50 & 34 & 49259 \\
\hline 87344 & 1.89 & 0.23 & 2.78 & 0.24 & 2.46 & 4.10 & 0.16 & 8.34 & 47 & 49321 \\
\hline 87887 & 2.11 & 0.08 & 2.96 & 0.12 & 3.83 & 3.74 & 0.07 & 8.47 & 8 & 49641 \\
\hline 92845 & 2.27 & 0.11 & 3.16 & 0.17 & 5.00 & 3.54 & 0.10 & 8.45 & 12 & 52407 \\
\hline 97585 & 2.17 & 0.10 & 3.03 & 0.16 & 4.26 & 3.66 & 0.08 & 8.47 & 13 & 54849 \\
\hline 101615 & 1.37 & 0.04 & 2.15 & 0.08 & 1.76 & 4.28 & 0.03 & 8.26 & 78 & 57013 \\
\hline 109573 & 1.37 & 0.04 & 2.18 & 0.10 & 1.68 & 4.33 & 0.03 & 7.05 & 126 & 61498 \\
\hline 114570 & 1.57 & 0.05 & 2.33 & 0.08 & 2.16 & 4.14 & 0.04 & 8.50 & 28 & 64466 \\
\hline 125473 & 2.17 & 0.06 & 3.06 & 0.10 & 3.84 & 3.76 & 0.04 & 8.43 & 7 & 70090 \\
\hline 129791 & 1.54 & 0.11 & 2.35 & 0.11 & 1.93 & 4.24 & 0.07 & 8.30 & 62 & 72192 \\
\hline 160263 & 2.32 & 0.16 & 3.25 & 0.20 & 5.02 & 3.55 & 0.12 & 8.41 & 13 & 86552 \\
\hline 170680 & 1.62 & 0.05 & 2.40 & 0.08 & 2.15 & 4.15 & 0.04 & 8.44 & 29 & 90806 \\
\hline 188228 & 1.48 & 0.02 & 2.31 & 0.09 & 1.74 & 4.32 & 0.02 & 7.43 & 111 & 98495 \\
\hline 193571 & 1.50 & 0.05 & 2.28 & 0.07 & 1.94 & 4.22 & 0.04 & 8.39 & 51 & 100469 \\
\hline 213320 & 1.90 & 0.09 & 2.72 & 0.12 & 2.87 & 3.96 & 0.07 & 8.48 & 12 & 111123 \\
\hline 213398 & 1.57 & 0.03 & 2.33 & 0.07 & 2.20 & 4.12 & 0.03 & 8.53 & 23 & 111188 \\
\hline 218639 & 1.48 & 0.09 & 2.27 & 0.09 & 1.92 & 4.23 & 0.07 & 8.37 & 60 & 114371 \\
\hline 223352 & 1.44 & 0.04 & 2.24 & 0.08 & 1.81 & 4.27 & 0.03 & 8.23 & 81 & 117452 \\
\hline 225200 & 1.69 & 0.10 & 2.45 & 0.11 & 2.44 & 4.05 & 0.08 & 8.53 & 21 & 345 \\
\hline
\end{tabular}

$\left(M_{V}\right)$ and their $\sigma$ are computed from the parallax data taken from the Hipparcos Main Catalogue and given in Cols. 13, 14 and 15, 16 of Table 2. These values are obtained by taking into account the colour excess.

One of our goals being to compute the stellar masses, we restrict the analysis to the reduced sample defined in the previous section.

We create the HR diagram by plotting the $T_{\text {eff }}$ derived from the MD calibration versus the luminosity (Fig. 9). The luminosity has been computed from $M_{V}$ with a bolometric correction taken from the Bessell et al. (1998) tables and $M_{\text {bol } \odot}=4.75$, as given by Cayrel de Strobel (1996), and adopted by IAU Commissions 29 and 36 (1997, IAU General Assembly). The values of $L / L_{\odot}$ are given in Table 4, Col. 2 and the errors in Col. 3; these latters are due to the errors on the parallaxes. On this plot the stars are divided according to their photometrically derived $\log g(\mathrm{MD})$ values. The evolutionary tracks by Schaller et al. (1992) are overplotted for 2.0, 2.5 and 3.0 solar masses, and $Z=0.02$.

The cross represents the Vega position $\left(T_{\text {eff }}=\right.$ $9550 \mathrm{~K} ; \log g=3.95$ according to Castelli \& Kurucz (1994). The position of the misclassified HD 216931 lies out of the boundaries of the plot.

We note the large spread in luminosity; we note also that Vega parameters do not represent the average values of dwarf A0 stars neither in luminosity nor in $T_{\text {eff }}$. 


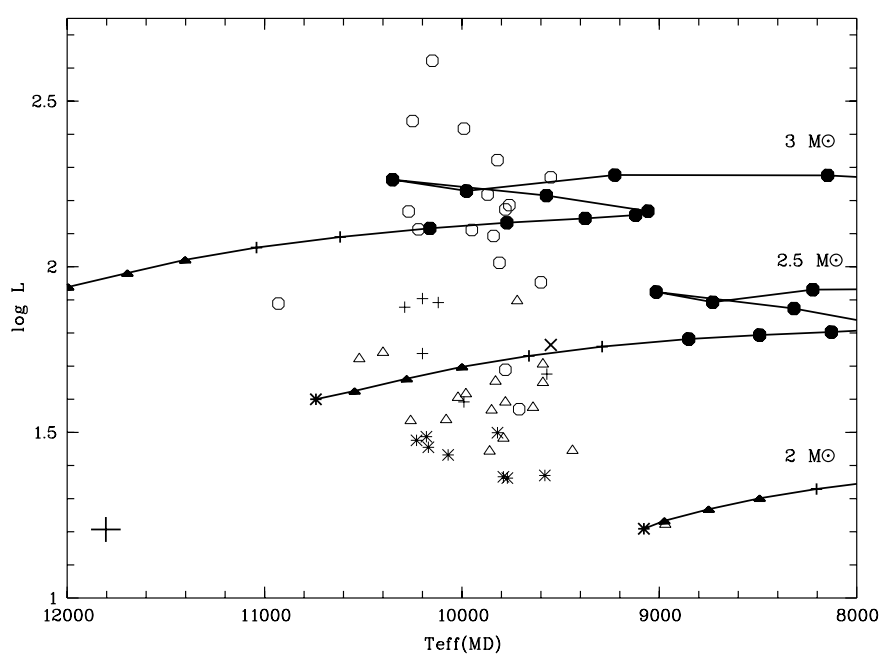

Fig. 9. The HR diagram for the reduced sample. The luminosity is expressed in $L / L_{\odot}$. The $\log g$ ranges are distinguished by using the following symbols: $* \log g \geq 4.3 ; \triangle 4.05 \leq \log g$ $<4.3 ;+3.8<\log g<4.05$; hexagone $\log g \leq 3.8$; The filled symbols triangle and hexagone are used for the evolutionary tracks with the same intervals for $\log g$. Vega is labelled with the symbol: $\times$

We remark that the only non peculiar A0 III star of our sample (HD 87887) does not have the lowest $\log g$ value (3.55), nor the brightest luminosity $\left(\log \left(L / L_{\odot}\right)=2.11\right)$. If we do not consider the shell stars, the most extreme values, which should indicate the most luminous objects, are the lowest value of $\log g=3.30$ (HD 67725) or the largest value for $\log \left(L / L_{\odot}\right)(2.62)$ (HD 85504); we note that both refer to stars classified as A0 V.

We recall that HD $67725\left(\log \left(L / L_{\odot}\right)=2.22\right)$ is reddened and has an $\mathrm{H}_{\gamma}$ profile which is not reproduced by computations. HD 85504 is a peculiar object which has the spectral properties of a Population I star and the kinematics of a Population II star, according to Adelman \& Pintado (1997).

\subsection{Evolutionary based surface gravity}

We used the HR diagram to derive the value of $\log g_{\mathrm{ev}}$ from evolutionary models and the Hipparcos parallaxes in order to compare them with the value of $\log g_{\mathrm{ph}}$ obtained from photometric data.

Balona (1994) found serious discrepancies between the gravities derived from evolutionary models and those obtained from photometric and spectroscopic determinations; this author questioned the reliability of the photometrically derived $\log g$ values and of the Kurucz (1979) LTE grid of models.

To compute $\log g_{\mathrm{ev}}\left(g_{\mathrm{ev}}=\mathrm{GM} / R^{2}\right)$, we applied the following relation:

$R=\left(L / 4 \pi \sigma T_{\text {eff }}^{4}\right)^{1 / 2}$

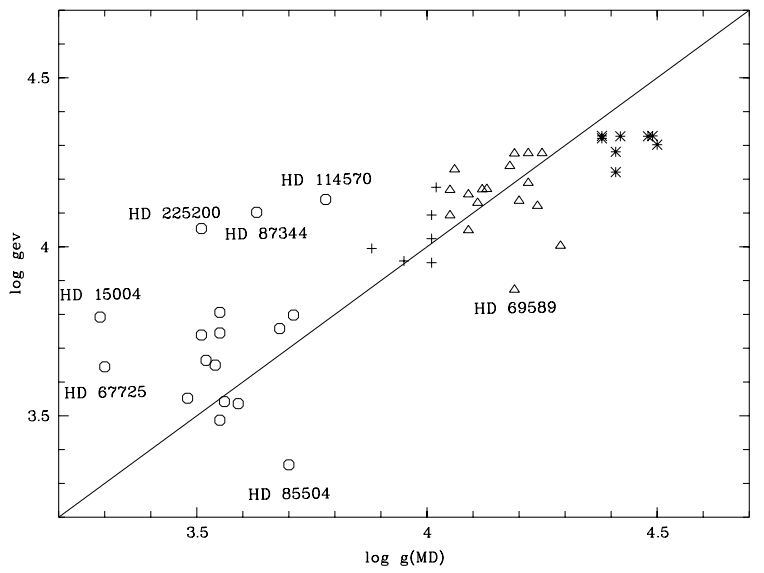

Fig. 10. The relation between $(\log g)_{\mathrm{ev}}$ and $(\log g)_{\mathrm{ph}}$; the latter is that derived from the MD calibration; the symbols are the same as in Fig. 9

The stellar masses $\mathcal{M} / \mathcal{M}_{\odot}$ are derived from $L / L_{\odot}$ and $T_{\text {eff }}$ by interpolation using Asiain (1998) method on the same evolutionary tracks (Schaller et al. 1992) used in the previous sub-section. The results are given in Table 4 Col. 4 and Col. 5 for the errors; the mean value of the error on the mass determination, due to the uncertainty on $M_{V}$ is $0.1 \mathcal{M} / \mathcal{M}_{\odot}$.

We compare these $\log g_{\text {ev }}$ (the values are given Table 4) with the $\log g_{\mathrm{ph}}$ obtained from $u b v y \beta$ photometric data Fig. 10. The $\log g_{\mathrm{ph}}$ have been already compared with model atmosphere calculations; our data include a Balmer line, which in this domain of temperature, is highly sensitive to the surface gravity. If we exclude the two shell stars (HD 15004, HD 225200) the most discrepent between the two $\log g$ determinations are for: HD 67725, HD 85504, HD 114570 whose peculiarities are discussed in the Appendix; for two more stars, HD 69589 and HD 87344, we do not have any obvious explanation.

From the diagram plotted Fig. 10 we note that, for most of the stars, the evolutionary gravities agree reasonably well with the photometric ones, the $\log g_{\mathrm{ev}}$ being systematically slightly higher than the $\log g_{\mathrm{ph}}$, except for the stars with $\log g \geq 4.3$. The behaviour of these "high" gravity stars is related to the systematic overestimation of $\log g$ by MD as discussed in Sect. 4 .

We cannot confirm, in our range of gravity values, the discrepancy found by Balona (1994), the discrepancy being, at least partly, due to the use of old Kurucz models and the Lester et al. (1986) synthetic indices.

\subsection{Mass-luminosity relation}

In order to evaluate the possible dependency of mass values with respect to the adopted stellar models, we compared the masses, as computed in the previous section, 
with those obtained by interpolating in the tracks by Bressan et al. (1993) and by Lebreton et al. (1997) using the CESAM code (Morel 1997). The differences between the masses computed from Schaller et al. (1992) and Bressan et al. (1993) have a mean value of -0.01 $\mathcal{M} / \mathcal{M}_{\odot}$ and for Lebreton computations, the differences have a mean value of $0.04 \mathcal{M} / \mathcal{M}_{\odot}$, which is negligible for our analysis.

We underline the fact that through the Hipparcos experiment the $\mathcal{M}$-L relationship can be derived from non eclipsing field stars. This relation is illustrated in Fig. 11a. We note the very tight relationship between the two parameters, mass and luminosity, but we recall that they cannot be considered as being totally independent both relying upon $M_{V}$ for their determination.

We have compared the relation derived from our sample of stars to the empirical one from binary systems (see Fig. 5 in Andersen 1991), also plotted in the same Fig. 11a. The general agreement appears clearly. Let us recall that our relation depends on stellar evolutionary models and that the relation based on eclipsing binaries is totally independent from a methodological point of view. Figure 11a shows that the gap around $\log \mathcal{M}=0.5$ in the Andersen sample is filled by the present study.

Andersen (1991) pointed out a significant scattering of the points around the mean relation and related it to age and chemical composition effects on luminosity. The evolutionary effect on the stars of our sample is revealed by the $\log g$ parameter. If we split the stars of our and Andersen samples (in the $T_{\text {eff }}$ range covered by A0 V stars) in narrow intervals of $\log g$ (see Fig. 11b), the dominant effect on the scatter is clearly related to gravity, since the mean relation for each group is smooth and better defined.

The use of the $\mathcal{M}$-L relationship to determine the mass of individual dwarf stars requires the knowledge of $\log g$, parameter decreasing with stellar age, even inside the stellar main sequence life as it can be seen Fig. 12 where the effect of evolution is emphasized by overplotting the values of mass and luminosity derived from evolutionary models. Only the points corresponding to the core hydrogen burning phase are displayed.

As a result the derived luminosity is directly related to the accuracy of the adopted $\log g$ value.

The masses extracted from an HR diagram $L-T_{\text {eff }}$ coupled with evolutionary tracks does not need the knowledge of $\log g$ and allows to derive the mass of a star by taking into account its distance from the ZAMS. In this case the uncertainty on the mass depends on the reliability of the evolutionary tracks.

\subsection{Ages}

The ages of the non binary and non peculiar stars have been determined by interpolation through the

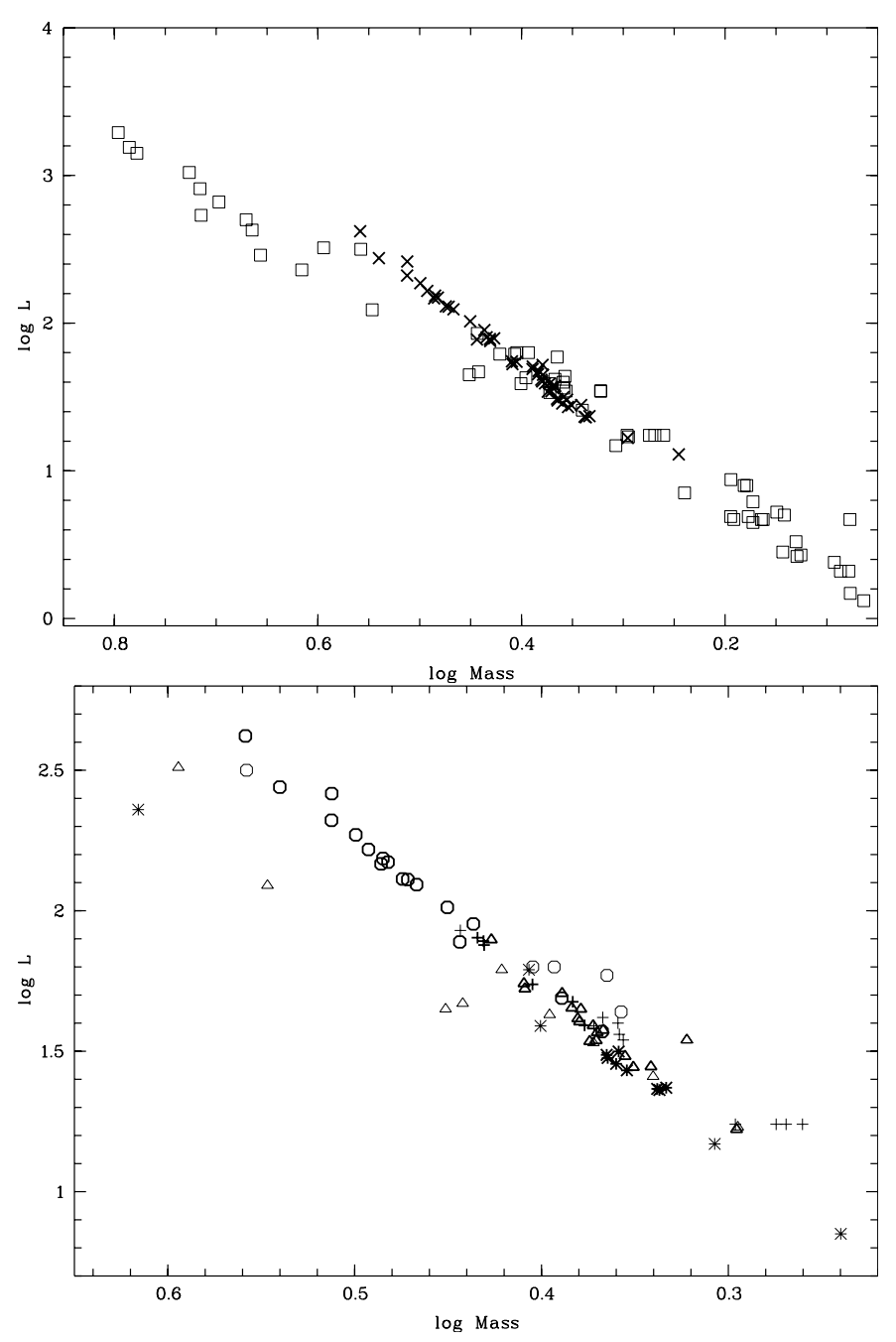

Fig. 11. a) The mass versus luminosity diagram for the stars of luminosity class $\mathrm{V}$ in our $(\times)$ and Andersen (square) samples. b) Zoom of the previous plot centered around A0 stars; the symbols are the same as in Fig. 9

evolutionary tracks in the HR diagram and are given in Col. 9 of Table 4, the errors are expressed in \% in Col. 10.

There are 6 stars younger than $10^{8}$ years.

From Table 4 and the corresponding Fig. 9 we note that a small number of objects are very near the ZAMS and may still preserve signs of their recent formation as, for instance, an IR excess. We underline once more that a significant number of slightly evolved stars is present among the A0 stars of luminosity class V.

\subsection{Rotational velocities}

We should not forget that a third parameter can affect the position of a star in the HR diagram: the rotational velocity. Such influence has been largely analysed from a theoretical point of view and recently studied, for stars hotter than those of the present paper, by Brown \& Verschueren (1997). 


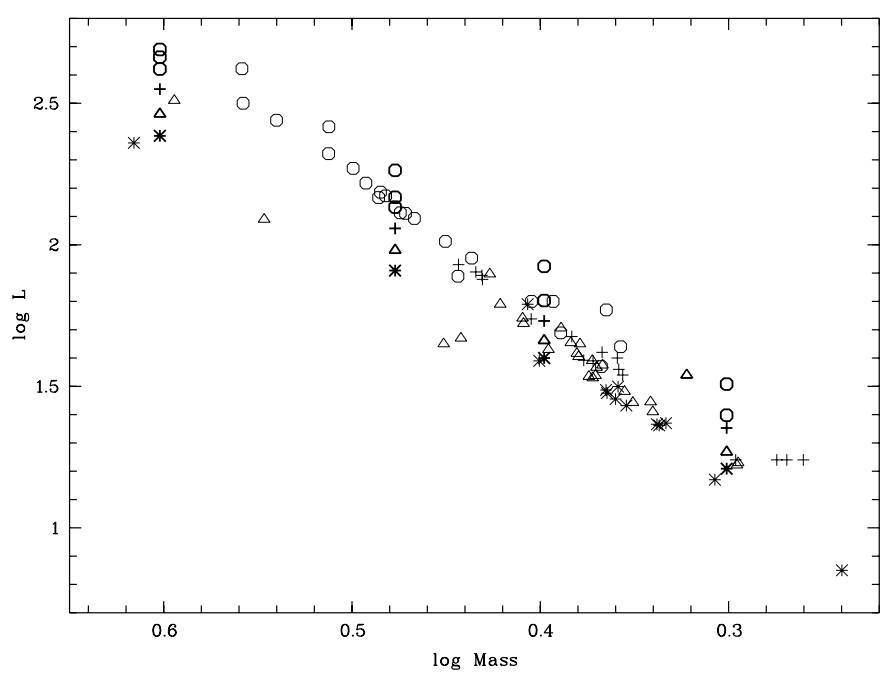

Fig. 12. The combined mass versus luminosity diagrams (same symbols as in Fig. 11b) on which the evolution of the luminosity for various masses (taken from Schaller et al. 1992, tables) is overplotted using the same symbols but with thick lines

We overplotted the observed spectra, after correction for RV, on the appropriate synthetic spectrum; the rotation value introduced in the computed spectrum that better fits the observations, is given in Col. 12 of Table 2, independent of the detection of established or suspected binarity. For some stars two values are given in the Table, when the $v \sin i$ was very uncertain; for HD 111786 the two values represent the velocities of the two components of the binary system. For the fastest rotators only $\mathrm{H}_{\gamma}$ and $\mathrm{Mg}$ II 4481 can be used for the comparison with computations.

We know that dwarf A-type stars are intrinsically fast rotators and generally characterized by mean-high values of $v \sin i$, with an average value not lower than $100 \mathrm{kms}^{-1}$. Stars with projected rotational velocities much lower than this value deserve some attention.

An account of the unknown parameter $i$, a low $v \sin i$ value may indicate that the object under study belongs to a class of the CP stars, is a close binary or is a Blue Horizontal Branch Star. From our sample we note that, among the 34 slowly rotating stars, 20 belong to one of the above categories.

The detection of binaries from broad lined spectra is more delicate and difficult; the availability of several orders of echelle spectra and the study of the cross correlation between observed and computed spectra has been fundamental for this detection.

Four of the observed stars belong to the Catalogue of $v \sin i$ standard stars by Slettebak et al. (1975). These stars are: HD 4150, HD 71155, HD 125473 and HD 188228.

\section{Conclusion}

Our aim was to define a set of "reference" dwarf A0 stars, having accurate parallaxes, in order to be combined with Vega in the role of "standard" stars.

The selection of non binary and non peculiar objects has been obtained from a selected sample of dwarf A0 extracted from Hipparcos Input Catalogue and observed spectroscopically. By combining photometric and spectroscopic informations with astrometric data and stellar evolutionary models, the original sample had to be reduced by $35 \%$.

The inclusion of a Balmer line in the analysis of stellar spectra of A-type stars is not trivial, owing the small size of the CCD detectors, but the informations that can be extracted are often underestimated. In a binary system, the contamination detected on the combined spectrum, when the two components belong to similar spectral types and have a different radial velocity, is expected first on Balmer lines since these are the strongest features over a large temperature interval. The second key line is the Mg II doublet at $4481 \AA$; its intensity is barely dependent on temperature and gravity for stars from late B to early A stars. A broadening of this line different from that expected by rotation can be detected with the resolution $R=28000$ in the spectra of stars with $v \sin i$ higher than $15 \mathrm{kms}^{-1}$.

In the original sample of non evolved A-type stars, we have detected a fairly large number of new binary stars, as well as suspected ones (Sects. 5 and 6); we also have detected peculiar objects such as a shell star, hot Am stars and a misclassified object.

The masses and luminosities derived for the reduced sample of stars which can be considered as "normal" has allowed to fill up the gap of the classical $\mathcal{M}$-L relation obtained from eclipsing binaries and to point out the important role of accurate $\log g$ values to define this relation (Sect. 8). The agreement found between the evolutionary based surface gravity $\log g_{\mathrm{ev}}$ and the value of $\log g_{\mathrm{ph}}$ obtained from photometric data does not confirm the discrepancies found by Balona (1994) at least in our gravity range.

Independently of $\log g$, the masses can be derived directly from the $L-T_{\text {eff }}$ HR diagram, but in this case the reliability of the evolutionary tracks becomes crucial.

In conclusion the two methods remain valid and complementary tools to determine the stellar masses.

Acknowledgements. The implementation of the Kurucz programs and the friendly help in using them by F. Castelli at Trieste has been highly appreciated. We would also thank P. Bonifacio for helpful suggestions and advices. We acknowledge A. Sellier for her technical assistance. Use was made of the SIMBAD data base, operated at the CDS, Strasbourg, France. Grants from MURST $40 \%$ and $60 \%$ are acknowledged. 


\section{Appendix}

HD 3003 Short period binary; both components are of A-type, according to the BSC. An IR excess has been measured from IRAS data by Stencel \& Backman (1992) and by Oudmaijer et al. (1992) who derived the [12]-[25] colour index $=-1.15$.

HD 4065 The spectra of the two components are A0 and A9, according to the BSC; the TD1 colours confirm that the secondary does not affect the UV colours. Recent speckle measures are given by Hartkopf et al. (1996) and by Horch et al. (1996).

HD 4150 The fit of the observed to the computed spectrum is such that this object is suspected to be a binary. Standard star for rotational velocity (Slettebak et al. 1975), but the $v \sin i=105 \mathrm{~km} \mathrm{~s}^{-1}$ value does not agree with that derived by (Levato 1972) $v \sin i=60 \mathrm{~km} \mathrm{~s}^{-1}$.

HD 7916 The peculiar spectrum is discussed in the text. The B component is classified $\mathrm{A} 8 \mathrm{p}$ ? and its peculiarities ascribed to contamination by the brighter companion by Corbally (1984) who gives, for the $V$ magnitudes of the components, $V_{\mathrm{A}}=6.42$ and $V_{\mathrm{B}}=8.26$.

HD 15004 Known shell star with a variable RV of the shell (Levato et al. 1995). Evidence of accreting, circumstellar gas from IUE spectra is discussed by Grady et al. (1996).

HD 21473 We note that among stars of our sample this is the "normal" star with the lowest $T_{\text {eff }}$ value. The star has been tested for photometric variability with negative result by Jorgensen et al. (1971) and confirmed by Hipparcos photometric data.

HD 34968 Slight variability of spectral lines noted by Gray \& Garrison (1987) on low resolution spectra. Photometric variable $V=4.67-4.72$ (SIMBAD data base), while no photometric variability was detected by Jorgensen et al. (1971) and confirmed by Hipparcos photometric data. Old MK classifications give A0 V var.

The Mg II 4481 is slightly asymmetric on the two spectra at our disposal.

HD 38206 The abundance analysis has been made by Lemke (1989 and 1990); the abundances of N and S have been added by Rentzsch-Holm (1997).

HD 45557 (ESA 340) Large differences between the $T_{\text {eff }}$ values derived from various indices have been found by Böhm-Vitense (1982), using visual and UV data. The other stars, in this paper, with similar $T_{\text {eff }}$ discrepancies are close binaries or shell stars.
HD 63112 This star has a low $\log g$ value for its A0 $\mathrm{V}$ spectral classification. A search for binarity by speckle interferometry gave negative results.

HD 67725 The core of $\mathrm{H}_{\gamma}$ is too narrow for the $250 \mathrm{~km} \mathrm{~s}^{-1}$ (in agreement with the $v \sin i=240 \mathrm{~km} \mathrm{~s}^{-1}$ by Dworetsky 1974) fitted by the Mg II 4481 profile. The $\log g=3.30$ derived from the MD procedure is the lowest of the sample and it is too low to fit the $\mathrm{H}_{\gamma}$ profile. Slight differences of $\mathrm{Mg}$ II 4481 profiles are detected on the two observed spectra.

HD 69589 An IRAS colour excess at $12 \mu \mathrm{m}$ has been derived by Patten \& Willson (1991).

HD 71043 Possible variability of the RV and large rotational velocity are noted by Andersen \& Nordström (1983).

HD 71155 Standard star for $v \sin i$ (Slettebak et al. 1975). The IR excess detected from IRAS at $25 \mu \mathrm{m}$ is interpreted as due to a dust shell (Coté 1987). In the UV, the $\mathrm{m}_{1565}$ is quite high compared with that of other stars of similar $T_{\text {eff }}$ and to the computed colours; the UV flux below $2500 \AA$ agrees with $T_{\text {eff }}=10000 \mathrm{~K}, 250 \mathrm{~K}$ higher than that obtained from visual data. The four spectra taken by us show some peculiar line profiles.

HD 79108 An IR excess at $60 \mu \mathrm{m}$ is derived from IRAS data (King 1994); the star is a $\lambda$ Boo candidate, according to Maitzen \& Pavlovski (1989).

HD 84461 We did not detect variations on the 3 spectra taken by us. The best fit with computations requires a lower $T_{\text {eff }}(9500 \mathrm{~K})$ than that derived from photometric colour indices.

HD 85504 The intriguing properties of this object are summarized by Cacciari (1985). An overabundance of $\mathrm{He}$ and $\mathrm{C}$ with respect to Sirius has been derived by Wallerstein et al. (1962) who formulate the hypothesis of a composite spectrum by a B8 and an A1 star.

Many metal lines are stronger in the observed, compared to the computed spectrum. We found many, but slight and difficult to prove, indications of binarity (e.g. the $\mathrm{H}_{\gamma}$ profile does not fit well those computed with the photometrically derived parameters; an abnormally large strength of many metal lines; the not rotationally broadened Mg II 4481 profile; the UV flux below $2500 \AA$ fits that computed with $T_{\text {eff }}=10500 \mathrm{~K}$, while the $m_{2740}-V$ suggests $\left.T_{\text {eff }}=10000 \mathrm{~K}\right)$.

The spectral peculiarities are studied by Adelman \& Pintado (1997).

HD 87344 The abundance analysis of this narrow-line star has been made by Lemke (1989 and 1990) and by 
Rentzsch-Holm (1997). The other component of this visual system, HD 87330, has been recognized to be a SB2; it is identified as HD 87344(2) in the HM Catalog and its photometric values are not correct (see Text).

HD 92845 The TD1 colours are better fitted by undereddened colours.

HD 109573 Star of $\beta$ Pic type with a very strong disk luminosity, even higher than that of $\beta$ Pic itself; a recent discussion of this young object can be found in Mouillet et al. (1997). The spectrum is characterized by weak metal lines; in particular, the Mg II 4481 profile is weak and not rotationally broadened and the UV colours (Fig. 8a) do not fit those predicted with the atmospheric parameters given in Table 2. Moreover, the $\log g=4.49$ derived from uvby $\beta$ photometry is too high for an A0 dwarf and does not fit the observed $\mathrm{H}_{\gamma}$ profile which better agrees with the $\log g=4.30$ derived from the parameters obtained from the Geneva undereddened colour indices. The Napiwotzki et al. (1993) correction of the gravity determined by the MD programs gives $\log g=4.43$, value that does not improve significantly the fit with the spectrum computed with the parameters derived from $u v b y \beta$ colours.

HD 111519 The cross correlation has a low and flat central part; the Mg II 4481 has a composite profile with a sharp and a broad component, signature of a double-lined spectroscopic binary; the metal lines present an important veiling effect.

HD 111786 Binary $\lambda$ Boo star discussed in the text and in Faraggiana et al. (1997). Among the stars of the present sample, this star has the lowest value of the cross correlation coefficient.

HD 114570 It has a very high colour excess, the $\mathrm{H}_{\gamma}$ profile is peculiar; it core suggests a $v \sin i$ lower than that obtained from metal lines and the UV dereddened colours fit better a $T_{\text {eff }}$ lower by $250 \mathrm{~K}$ than that derived from visual data.

HD 125473 This star is classified A0 III by Gray \& Garrison (1987). It is a dusty system on the basis of IRAS data (Cheng et al. 1992). We did not detect differences between the 5 spectra at our disposal. This is the star for which the highest difference in $T_{\text {eff }}$ between MD and Geneva calibrations has been found; the $\mathrm{H}_{\gamma}$ profile is fitted by the spectrum computed with the MD parameters.

HD 129791 It is an X-ray source (Schmitt et al. 1993) difficult to explain since A0-type stars are not X-ray emitters. A relatively high dereddening is requested to reproduce the colours of a normal star. The lines are extremely broadened and their profile suggest a composite spectrum with different $v \sin i$ of the two components.
HD 139129 The abundance analysis has been made by Lemke $(1989,1990)$ who adopted $T_{\text {eff }}=9900 \mathrm{~K}$ and $\log g=3.7$ and derived non solar abundances for several elements. Further discussion on the metal abundances is in Rentzsch-Holm (1997). According to Jaschek et al. (1991), this star has an unexplained IR excess at $12 \mu \mathrm{m}$.

HD 151527 No meaningful $M_{V}$ can be computed owing the high distorted colours from which large and discrepant colour excesses are derived (see Table 2). The peculiarity of the visual and UV colours and of the spectrum suggests that this object is more complex than a simple binary star. The UV TD1 colours are very distorted.

HD 152849 According to Richichi et al. (1997) discussion of this star, the companion has also a spectral type A and is only slightly later than the primary. Recent speckle measures are in Horch et al. (1996).

HD 188228 The $v \sin i$ value measured by Slettebak et al. (1975) $\left(v \sin i=80 \mathrm{~km} \mathrm{~s}^{-1}\right)$ does not agree with that obtained by Levato (1972) $\left(v \sin i=140 \mathrm{~km} \mathrm{~s}^{-1}\right)$. A variability is mentioned in old MK classifications.

The Mg II profile is not rotationally broadened; several metal lines have a flat core and the UV fits better $T_{\text {eff }}=10500 \mathrm{~K}$.

HD 193571 It is a protoplanetary system candidate on the basis of the IR excess detected by IRAS (Cheng et al. 1992); no signatures of CS features have been observed by Holweger \& Rentzsch-Holm (1995).

HD 213320 It belongs to the hot extension of the Am sequence according to Adelman \& Nasson (1980), but with several abundance anomalies (Adelman et al. 1984); it is an SB according to Dworetsky (1974).

HD 225200 Ambiguous classifications are found in the literature: it is a binary $\mathrm{A} 0 \mathrm{~V}+\mathrm{A}$ : according to Levato et al. (1979) and B9 IVs + A2n according to AM, but A0 $\mathrm{IV}^{-}$(shell) according to Gray \& Garrison (1987).

\section{References}

Abt H.A., Morrell N.I., 1995, ApJS 99, 135 [AM]

Adelman S.J., Nasson M.A., 1980, PASP 92, 346

Adelman S.J., Young J.M., Baldwin H.E., 1984, MNRAS 206, 649

Adelman S.J., Pintado O.I., 1997, A\&AS 125, 219

Andersen J., 1991, A\&AR 3, 91

Andersen J., Nordström B., 1983, A\&AS 52, 471

Asiain, 1998, Ph Thesis Universitat de Barcelona

Balona L.A., 1994, MNRAS 268, 119

Bessell M.S., Castelli F., Plez B., 1998, A\&A 333, 231 and 337, 321 
Bressan A., Fagotto F., Bertelli G., Chiosi C., 1993, A\&AS 100,647

Böhm-Vitense E., 1982, ApJ 255, 191

Brown A.G.A., Verschueren W., 1997, A\&A 319, 811

Burnage R., Gerbaldi M., 1990, 2nd ESO/ST-ECF Data Analysis Workshop, Baade D., Grøsbol P. (eds.). ESO Garching, p. 137

Burnage R., Gerbaldi M., 1992, 4nd ESO/ST-ECF Data Analysis Workshop, Grøsbol P., de Ruijsscher R.C.E. (eds.). ESO Garching, p. 159

Cacciari C., 1985, A\&AS 61, 407

Castelli F., 1991, A\&A 251, 106

Castelli F., Kurucz R.L., 1994, A\&A 281, 817

Cayrel de Strobel G., 1996, A\&AR 7, 243

Cheng K.P., Bruhweiler F.C., Kondo Y., Grady C.A., 1992, ApJS 396, L83

Collins G.W., II, Smith R.C., 1985, MNRAS 213, 519

Corbally C.J., 1984, ApJS 55, 657

Corbally C.J., 1987, ApJS 63, 365

Coté J., 1987, A\&A 181, 77

Dworetsky M.M., 1974, ApJS 28, 101

Dworetsky M.M., Moon T.T, 1986, MNRAS 220, 787

ESA, The Hipparcos Catalogue, 1997, SP-1200

Faraggiana R., Gerbaldi M., Burnage R., 1997, A\&A 318, L21

Figueras F., Torra J., Jordi C., 1991, A\&AS 87, 319

Gerbaldi M., Gómez A.E., Faraggiana R., et al., 1989, The Messenger 56, 12

Gerbaldi M., Faraggiana R., Burnage R., et al., 1998a, Contrib. Astron. Obs. Skalnaté Pleso 27, 197

Gerbaldi M., Faraggiana R., Burnage R., et al., 1998b, IAU Highlights (in press)

Grady C.A., Pérez M.R., Talavera A., et al., 1996, ApJ 471, L49

Gray R.O., Garrison R.F., 1987, ApJS 65, 581

Hartkopf W.I., Mason B.D., McAlister H.A., et al., 1996, AJ 111,936

Hauck B., Mermilliod M., 1990, A\&AS 86, 107 [HM]

Hoffleit D., Jaschek C., 1982, Bright Star Catalogue, Yale University Observatory [BSC]

Hoffleit D., Warren W.H., 1994, The Bright Star Catalogue, 5th revised ed., version 1994 June (private communication) [BSC1994]

Holweger H., Rentzsch-Holm I., 1995, A\&A 303, 819

Horch E.P., Dinescu D.I., Girard T.M., et al., 1996, AJ 111, 1681

Jaschek C., Jaschek M., Andrillat Y., Egret D., 1991, A\&A 252,229

Jaschek C., Valbousquet A., 1997, A\&AS 126, 251
Jordi C., Masana E., Figueras F., et al., 1997, A\&AS 123, 83 Jorgensen H.E., Johansen K.T., Olsen E.H., 1971, A\&A 12, 223

King J.R., 1994, MNRAS 269, 209

Künzli M., North P., Kurucz R.L., et al., 1997, A\&AS 122, 51

Kurucz R.L., 1979, ApJS 40, 1

Kurucz R.L., 1993, CD Rom 13 and 18

Lebreton Y., Perrin M.-N., Fernandes J., et al., 1997, ESA SP-402, 379

Lemke M., 1989, A\&A 225, 125

Lemke M., 1990, A\&A 240, 331

Lester J.B., Gray R.O., Kurucz R.L., 1986, ApJS 61, 509

Levato O.H., 1972, PASP 84, 584

Levato O.H., Morrell N.I., Malaroda S., 1979, Rev. Mex. A. A. 4,321

Levato O.H., Mallaroda S., Jaschek C., Jaschek M., 1995, A\&A 299,163

Maitzen H.M., Pavlovski K., 1989, A\&AS 81, 335

Mermilliod J.-C., Mermilliod M., Hauck B., 1997, A\&AS 124, 349

Moon T.T., 1985, Comm. from the Univ of London Obs. 78 and Revisions 1985 (private communication)

SA Moon T.T., Dworetsky M.M., 1985, MNRAS 217, 305 [MD]

Morel P.J., 1997, A\&AS 124, 597

Mouillet D., Lagrange A.M., Beuzit J.L., Renaud N., 1997, A\&A 324, 1083

Napiwotzki R., Schönberner D., Wenske V., 1993, A\&A 268, 653

Oudmaijer R.D., van der Veen W.E.C.J., Waters L.B.F.M., et al., 1992, A\&AS 96, 625

Patten B.M., Willson L.A., 1991, AJ 102, 323

Rentzsch-Holm I., 1997, A\&A 317, 178

Richichi A., Calamai G., Leinert Ch., et al., 1997, A\&A 322, 202

Schaller G., Schaerer D., Meynet G., et al., 1992, A\&AS 96, 269

Schmitt J.H.M.M., Zinneker H., Cruddace R., Harnden F.R. Jr., 1993, ApJ 402, L13

Slettebak A., Collins II G.W., Boyce P.B., et al., 1975, ApJS 129, 137

Slettebak A., Kuzma T.J., Collins G.W. II, 1980, ApJ 242, 171

Smalley B., Dworetsky M.M., 1993, A\&A 271, 515

Stencel R.E., Backman D.E., 1991, ApJS 75, 905

Strömgren B., 1966, ARA\&A 4, 433

Turon C., Crézé M., Gómez A., et al., 1992, The Hipparcos Input Catalogue, ESA SP-1136

Thompson G.I., Nandy K., Jamar C., et al., 1978, Catalogue of Stellar Ultraviolet Fluxes The Science Research Council

Wallerstein G., Stone Y.H., Williams J.A., 1962, ApJ 135, 459 